\title{
GeoNet: An open source software for the automatic and objective extraction of channel heads, channel network, and channel morphology from high resolution topography data
}

\author{
Harish Sangireddy ${ }^{\mathrm{a}}$, Colin P. Stark ${ }^{\mathrm{b}}$, Anna Kladzyk ${ }^{\mathrm{a}}$, Paola Passalacqua ${ }^{\mathrm{a}}$ \\ ${ }^{a}$ Department of Civil, Architectural and Environmental Engineering, and Center for \\ Research in Water Resources, The University of Texas at Austin, Austin, TX \\ ${ }^{b}$ Lamont-Doherty Earth Observatory, Columbia University, NewYork, USA.
}

\begin{abstract}
Extracting hydrologic and geomorphic features from high resolution topography data is a challenging and computationally demanding task. We illustrate the new capabilities and features of GeoNet, an open source software for the extraction of channel heads, channel networks, and channel morphology from high resolution topography data. The method has been further developed and includes a median filtering operation to remove roads in engineered landscapes and the calculation of hillslope lengths to inform the channel head identification procedure. The software is now available in both MATLAB and Python, allowing it to handle datasets larger than the ones previously analyzed. We present the workflow of GeoNet using three different test cases; natural high relief, engineered low relief, and urban landscapes. We analyze default and user-defined parameters, provide guidance on setting parameter values, and discuss the parameter effect on the extraction results. Metrics on computational time versus dataset size are also presented. We show the ability of GeoNet to objectively and accurately extract channel features in terrains of various characteristics.
\end{abstract}

Keywords: Lidar, high resolution topography, geodesics, channel heads, channel network, channel morphology

Preprint submitted to Environmental Modelling $\mathcal{E}$ Software

April 29, 2016

(C) 2016. This manuscript version is made available under the Elsevier user license http://www.elsevier.com/open-access/userlicense/1.0/ 


\section{Software}

$\begin{array}{ll}\text { Name } & \text { GeoNet } \\ \text { Developer } & \text { GeoNet team } \\ \text { Contact } & \text { https://sites.google.com/site/geonethome/ } \\ \text { Software required } & \text { The MATLAB version of GeoNet requires: Im- } \\ & \text { age Processing Toolbox, Mapping Toolbox, and } \\ & \text { Statistics Toolbox. A version of GeoNet combined } \\ & \text { with gdal libraries is available for use without the } \\ & \text { Mapping Toolbox. The Python version of GeoNet } \\ & \text { requires: GRASS GIS, Python libraries NumPy, } \\ & \text { scikit-fmm, and SciPy. } \\ & \text { https://sites.google.com/site/geonethome/ } \\ & \text { source-code } \\ \text { http://opentopo.sdsc.edu/tools/listTools }\end{array}$

\section{Introduction}

Channels are an integral part of the Earth system topography and morphology as they transport water, solids, and solutes from upstream regions to the coast, support habitat for various species, and are critical sources of fresh water. The identification of these transport pathways is essential, for example, for understanding the hydrologic cycle, land surface-atmosphere interactions, and the Earth surface response to environmental change.

Several methods have been proposed to extract channel networks from digital elevation models (DEMs) (e.g., Montgomery and Dietrich, 1988; Tarboton et al., 1988; Montgomery and Foufoula-Georgiou, 1993; Costacabral and Burges, 1994; Giannoni et al., 2005; Hancock and Evans, 2006). Commonly, these methods follow a work-flow consisting of: (a) DEM pit filling, (b) flow direction assignment (either using the D8 or Dinfinity method (Tarboton, 1997)), (c) computation of flow accumulation, (d) identification of catchment boundaries, and of (e) catchment outlets, and (f) extraction of channels based on a flow accumulation threshold or a combination of area, slope, and/or terrain curvature (e.g., Tarboton and Ames, 2001; Lacroix et al., 2002; Orlandini et al., 2003; Tesfa et al., 2011; Sofia et al., 2011; Yang et al., 2014; Cazorzi et al., 2013; Sofia et al., 2014a,b; Bhowmik et al., 2015). This work-flow is common to methods that use a raster dataset as input and differs from work-flows used to analyze triangulated irregular networks (e.g., Jones et al., 1990; Nelson et al., 1994; Vivoni et al., 2004; Zhou 
et al., 2011) and extract networks from contour elevation data (e.g., Moretti and Orlandini, 2008).

The explosive growth of high resolution data acquisition systems (e.g., airborne, terrestrial, mobile laser scanning systems) and data dissemination applications and related services (e.g., National Elevation Dataset http: //viewer.nationalmap.gov/viewer/ and OpenTopography http://www . opentopography.org/) are generating petabytes of data. The vast spatial extents and fine spatial resolutions allow us to map landscape features (e.g., rivers, floodplains, landslide scars) and improve the understanding of the underlying geomorphic processes at scales over which such processes act, transforming the way we analyze landscapes (Tarolli and Dalla Fontana, 2009; Glennie et al., 2013; Passalacqua et al., 2014; Tarolli, 2014; Passalacqua et al., 2015; Harpold et al., 2015).

Improved data, though, require improved analysis methods. When applied to high resolution topography data, in fact, the classic channel network extraction work-flow described above has some shortcomings, e.g., the pit filling operation, which alters the original input DEM and can remove important land surface information and the definition of a flow accumulation threshold, which is based on the assumption that all channels begin at the same flow accumulation value, which is usually not valid (McNamara et al., 2006). Recently developed methods for the extraction of channel heads and channel networks from high resolution topography data (e.g., Lashermes et al., 2007; Passalacqua et al., 2010a; Orlandini et al., 2011; Tesfa et al., 2011; Pelletier, 2013) are able to map channel networks at the correct drainage density by avoiding the strict use of a flow accumulation threshold.

One of these methods is GeoNet (Passalacqua et al., 2010a), an open source, automatic method for channel head, channel network, and channel morphology extraction from high resolution topography data. The method combines nonlinear filtering of elevation data, to smooth small scale variability and enhance features of interest, a statistical analysis of curvature, to identify the set of likely channelized pixels, and geodesic minimization principles to extract channel heads and channel centerlines. GeoNet has been applied and tested on a steep and natural landscape (Passalacqua et al., 2010a), a heterogeneous mountainous watershed (Passalacqua et al., 2010b), and a flat and engineered landscape (Passalacqua et al., 2012). The method was coded in MATLAB and C, with limitations to the size of input datasets.

In this paper, with the aid of three study sites representing natural (Tennessee Valley, CA), flat and engineered (Le Sueur River, MN), and urban 
landscapes (Little Walnut Creek, TX) (Section 2), we present the work-flow (Section 3) and the new capabilities of GeoNet (method and software). The most recent version of GeoNet has been improved in filtering natural and road features and in the identification of channel heads (Section 4). The code is available in MATLAB and Python and allows integration of software libraries for data access, algorithm development and integration with GIS software such as ArcGIS, QGIS, and GRASS GIS (Section 5). The workflow steps are described and tested on the study sites (Section 6) and metrics of computational time versus input dataset size are provided. A discussion of the results and the conclusions of this work are in Section 7.

\section{Study sites}

\subsection{Natural landscape: Tennessee Valley, $C A$}

The Tennessee Valley catchment lies north of San Francisco in Marin County, California (Figure 1). This catchment has been studied extensively by previous authors; of particular interest for our application is work done on channel initiation and the availability of field surveyed channel heads and channel network (Montgomery and Dietrich, 1988, 1989; Dietrich et al., 1993). The terrain is characterized as mostly soil mantled, with local bedrock outcrops on ridges and on steep slopes. High resolution topography data at $1 \mathrm{~m}$ resolution are available (http://www. opentopography.org/). 
(a)

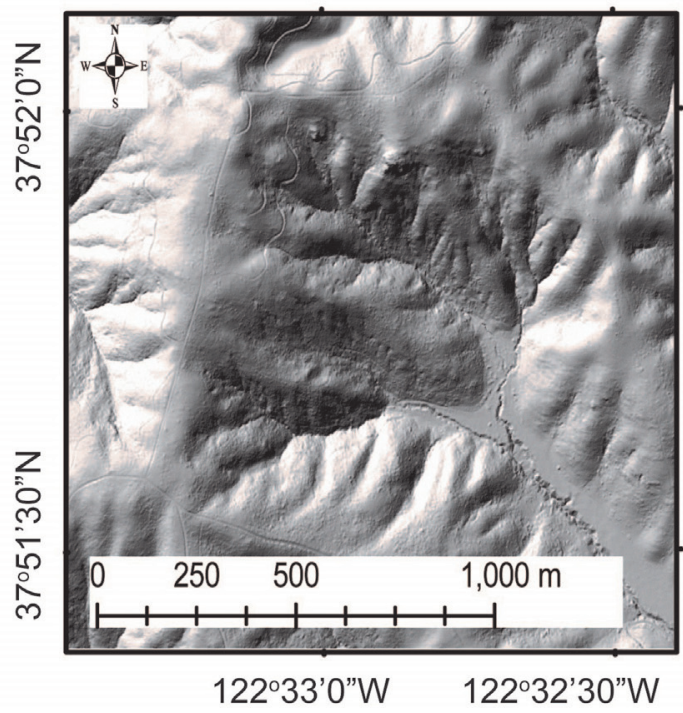

(b)

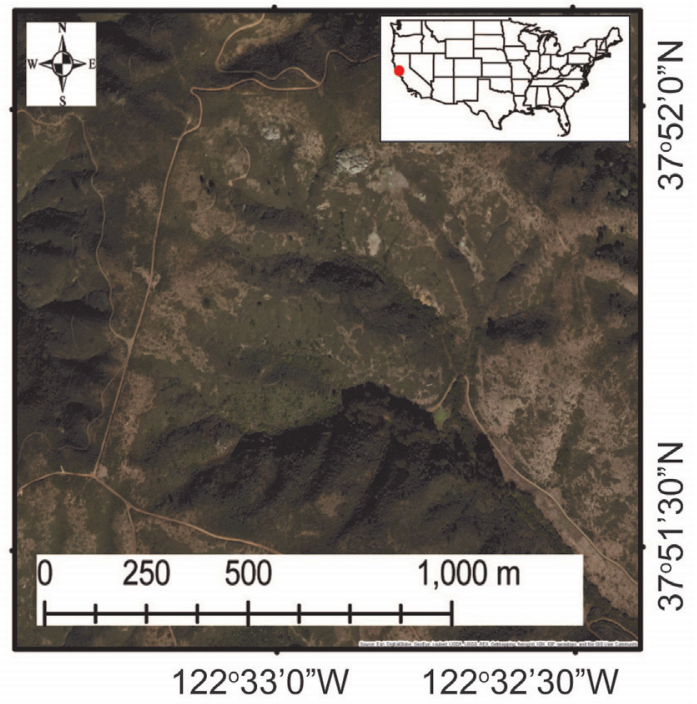

Figure 1: Tennessee Valley, CA, a natural and high relief landscape. (a) Hillshade prepared in ArcGIS, and (b) satellite imagery published in Esri's World Imagery map available as basemap in ArcGIS Desktop.

\subsection{Flat and engineered landscape: Le Sueur River Basin, $M N$}

The Le Sueur River Basin is located in southern Minnesota (Figure 2). It has been extensively studied as it is a major source of sediment to the Minnesota River (Gran et al., 2009; Belmont et al., 2011; Gran et al., 2011). Due to the presence of natural channels, artificial drainage ditches, roads, and overall small topographic gradients, the watershed poses several challenges to classic feature extraction algorithms (Passalacqua et al., 2012). High resolution topography data are available (Gran et al., 2009, 2011; Belmont et al., 2011). The data used here are at $3 \mathrm{~m}$ resolution. 
(a)

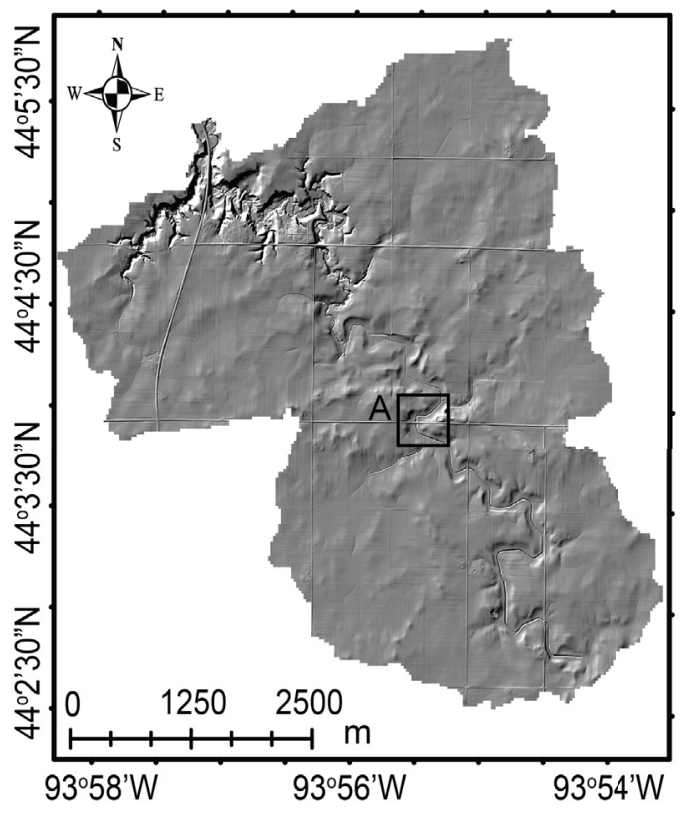

(b)

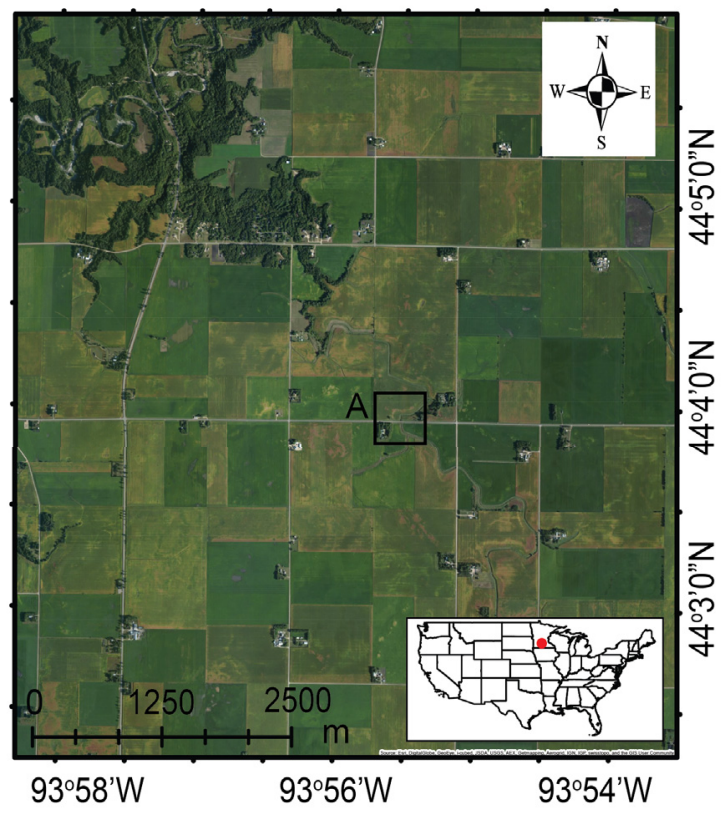

Figure 2: Le Sueur River Basin, MN, a low relief and engineered landscape. (a) Hillshade prepared in ArcGIS, and (b) satellite imagery published in Esri's World Imagery map available as basemap in ArcGIS Desktop.

\subsection{Urban landscape: Little Walnut Creek, TX}

Little Walnut Creek is located in Travis County and is part of an urban suburb of the larger metropolitan area of Austin, Texas (Figure 3). We chose this area as first application of GeoNet to an urban landscape. With the term 'urban' we indicate an engineered landscape that presents multiple features typical of an urban setting including stormwater collection systems such as curbs, gutters, and inlets (region A in Figure 3), roads (region B in Figure 3), and bridges (region $\mathrm{C}$ in Figure 3). These engineered features have modified the shape of the landscape and the flow of water, resulting in parallel surface and subsurface flow.

High resolution topography data was made available by the City of Austin as a classified point cloud file in LAS file format. The ESRI LAS Dataset Tools in ArcGIS were used to convert the points classified as ground to a 1 $m$ resolution raster grid. The building footprints for this region (Figure 3) were also obtained from the City of Austin. 
(a)

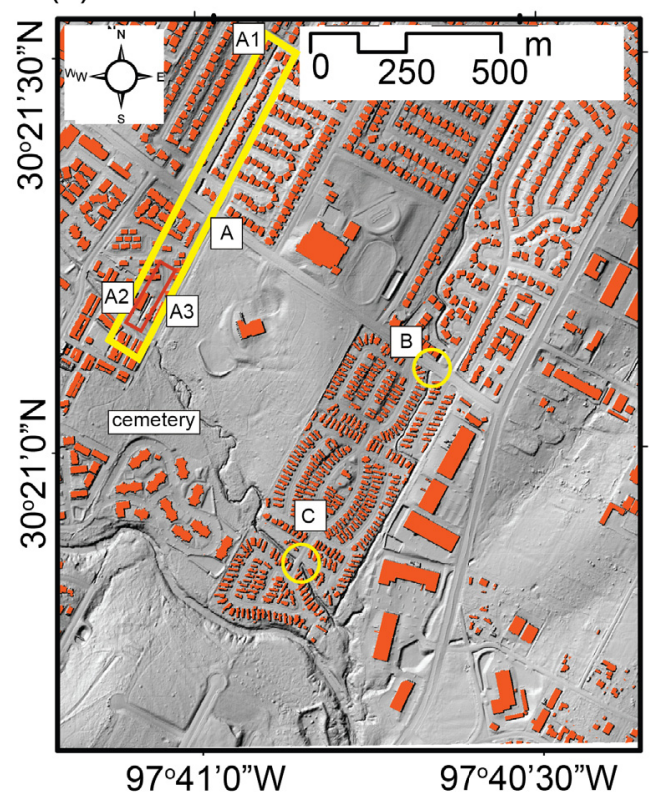

(b)

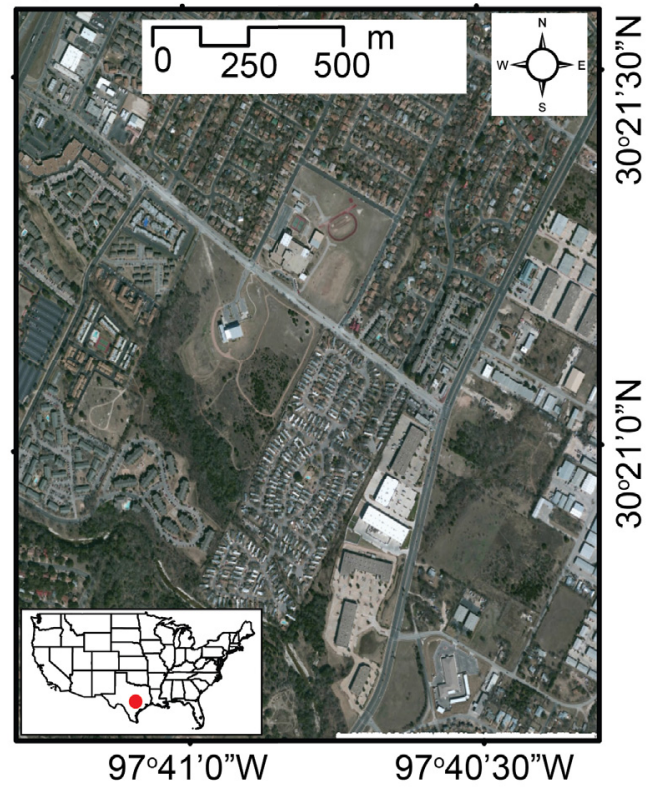

Figure 3: Little Walnut Creek, Austin, TX, an urban landscape. (a) Hillshade prepared in ArcGIS. Region A shows a storm collector that starts at A1, goes underground, and reappears on the surface at A2, upstream of the junction with Little Walnut Creek at A3. Region B shows a local drain intersected by a road. Region C shows Little Walnut Creek at the intersection with a bridge. The building footprints are overlaid in orange. (b) Satellite imagery published in Esri's World Imagery map available as basemap in ArcGIS Desktop.

\section{GeoNet work-flow}

The GeoNet method consists of three major components: (i) nonlinear filtering of the elevation data, (ii) identification of likely channelized pixels through the statistical analysis of curvature, and (iii) channel heads and channel network extraction based on geodesic minimization principles. The work-flow of GeoNet (Figure 4) begins by reading the input bare earth DEM. The mean slope of the raw input DEM is computed to define whether the terrain is steep (slope $\geq 5^{\circ}$ ) or flat (slope $\leq 5^{\circ}$ ) (McCullagh, 1978) (this slope value can be modified if desired). If the terrain is steep, the curvature is set to geometric, while in flat terrains the curvature is set to laplacian. 
Nonlinear filtering is applied to remove small scale variability. In engineered landscapes the nonlinear filtering operation follows the application of a median filter that removes features such as roads. Slope and curvature are computed on the filtered DEM and the qq-plot of curvature is used to identify the convergent features of the landscape and obtain the set of likely channelized pixels. This set of pixels represents a first estimate of the channel network. Upon computing flow accumulation, the skeleton of likely channelized pixels is refined based on the user-defined skeleton thinning parameter (Parameters. flowThresholdforSkeleton in the code) (Passalacqua and Foufoula-Georgiou, 2015).

The cost function is used to compute geodesic distances via the FastMarching algorithm. End point detection identifies channel heads by using the skeleton's connected components information, the geodesic distance, and a search box size equal to the median hillslope length. Finally, a gradient descent is performed on the geodesic distance from the channel heads to the basin outlet to extract the channel centerlines. 


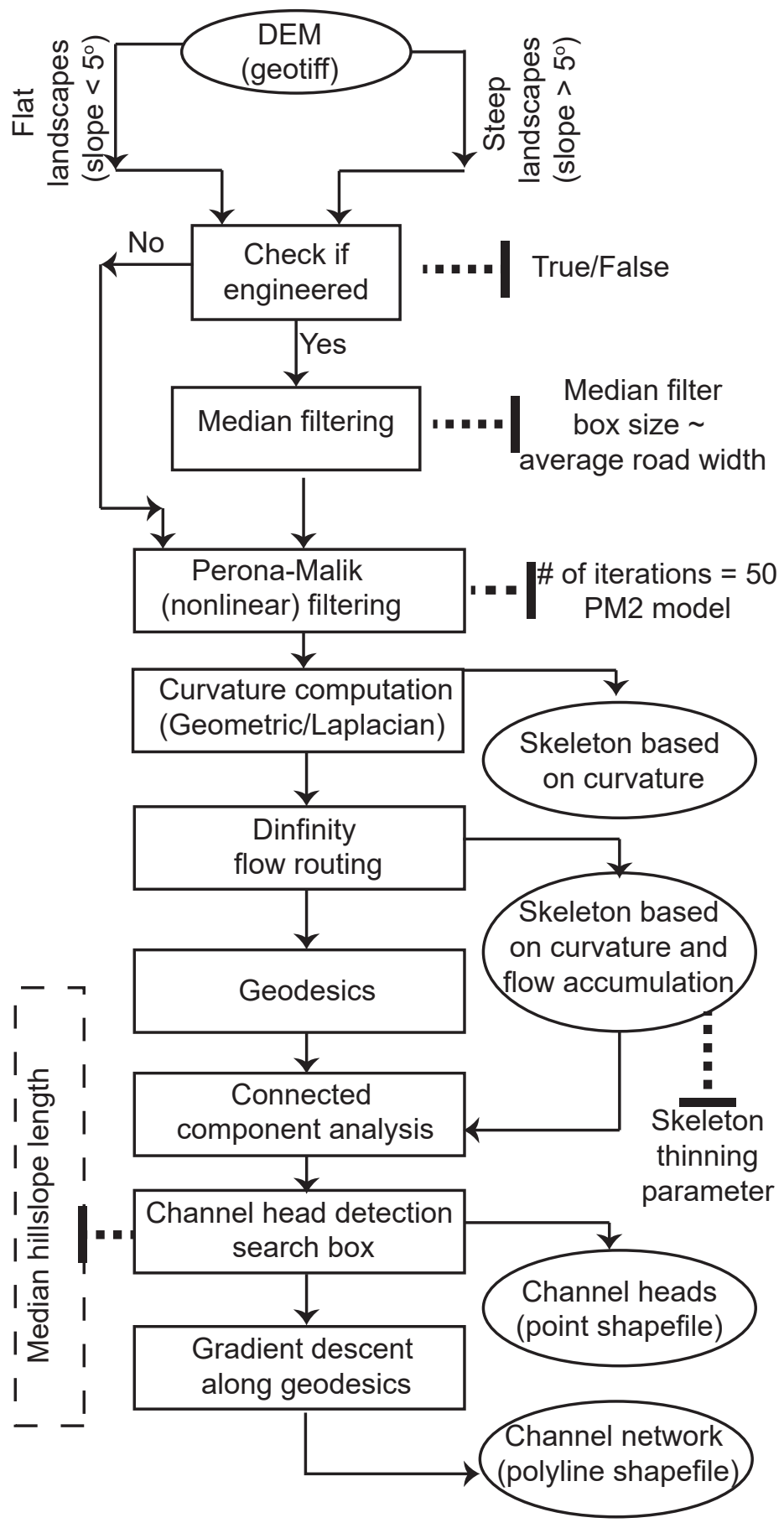

Figure 4: The work-flow of GeoNet. All input and output steps are in ellipses, while processing steps are in rectangles. 
Figure 4: (Continued..) Processing parameters are indicated by a dashed indent. The median hillslope length is computed within GeoNet. Only the skeleton thinning parameter and the median filter box size (in urban landscapes) are required as user inputs.

To run GeoNet, user input is required on the value of the skeleton thinning parameter. Additionally, in urban landscapes the use of median filtering to remove roads requires setting the filter box size to the average road size in the region of interest. The median filter box size is set to a default value of $21 \mathrm{~m}$.

In the next section we briefly describe the existing components of GeoNet and further explore aspects of filtering (edge-stopping function, number of filtering iterations) and of channel head detection (search box size) that have not been previously analyzed, as well as describe new capabilities of the method (computation of hillslope length and median filtering). We refer the reader to Passalacqua et al. (2010a,b, 2012) for detailed information on the existing portions of the method.

\section{Method development}

4.1. Filtering to remove small scale variability and enhance features of interest

When analyzing high resolution topography data with the goal of extracting features of interest, an operation of filtering is usually performed to regularize the elevation data. This operation consists of removing unwanted portions of the signal (i.e., small scale surface variability) while maintaining and enhancing features of interest for the problem at hand. For example, in the case of channel network extraction, we may want to filter out the bumpiness of the ground, while preserving features such as channel banks sharp and localized.

Several filtering techniques have been proposed (e.g., Wiener, 1949; Burt and Adelson, 1983; Koenderink, 1984; Witkin, 1984; Perona and Malik, 1990). A popular filter among them is the linear diffusion equation (Koenderink, 1984):

$$
\frac{\partial h(x, y, t)}{\partial t}=\nabla \cdot(c \nabla h)
$$

where $h(x, y, t)$ is the elevation data at time $t, c$ is the diffusion coefficient, and $\nabla$ is the gradient operator. The linear diffusion equation is isotropic as the diffusion coefficient $c$ is constant in space and time. While simple to 
apply, the main disadvantage of this method is that it may result in blurring of the feature edges with loss of sharpness and localization (Passalacqua et al., 2010b).

The diffusion filter (equation 1) may be configured to smooth irregularities (noise) while retaining the features of interest by making the diffusion coefficient $c$ a function of space and time. This operation results in a nonlinear diffusion equation as proposed by Perona and Malik (1990):

$$
\frac{\partial h(x, y, t)}{\partial t}=\nabla \cdot[c(x, y, t) \nabla h]
$$

Note that here a dimensionless form of the nonlinear diffusion equation has been used with a dimensionless diffusion coefficient $c$ and 'time' scaled by diffusion and expressed effectively in $L^{2}$ units. Two possible forms of the diffusion coefficient are (Perona and Malik, 1990):

$$
\begin{gathered}
c=e^{\left(-(|\nabla h| / \lambda)^{2}\right)} \\
c=\frac{1}{1+\left(\frac{|\nabla h|}{\lambda}\right)^{2}}
\end{gathered}
$$

where $|\nabla h|$ is the absolute value of the elevation gradient at location $x, y$ and time $t$ and $\lambda$ is the edge stopping threshold computed as the 90th quantile of the gradient distribution (Perona and Malik, 1990). Equations (3) and (4) are also called edge stopping functions and we will refer to them as PM1 (equation 3) and PM2 (equation 4) in the following. The physical basis for these functions is that gradients are high for feature boundaries (edges). Thus, PM1 and PM2 promote diffusion within feature boundaries and penalize it across them, preserving edges.

Stability issues of the Perona-Malik formulation were later identified and addressed by Catté et al. (1992). Their stable form of the nonlinear diffusion filter involves convolution of the landscape with a small Gaussian kernel at each time step. This form of the filter is implemented in GeoNet (Passalacqua et al., 2010a) with an explicit numerical scheme (Perona and Malik, 1990; Catté et al., 1992; Gilboa et al., 2001) in which the time step is set based on the Von Neumann stability criterion (Weickert, 1997):

$$
\delta t \leq \frac{1}{4}(\delta x)^{2}
$$


where $\delta x$ is the pixel size. Note that since time is in diffusion scaled $L^{2}$ units, equation (5) is dimensionally consistent. Our numerical solution sets $\delta t=$ $\frac{1}{4} \delta x^{2}$ and we refer to each numerical time step of the smoothing operation as an iteration.

\subsubsection{Analysis of the edge stopping function and time of forward diffusion}

The default edge stopping function in GeoNet is PM2 and the time of forward diffusion is set to a value of 50 iterations, however, an investigation of the effect of the edge stopping function on the results had not been performed and 50 iterations were adopted following the image processing literature. Here we establish quantitative metrics to inform the choice of the default edge stopping function and time of forward diffusion.

Mrázek and Navara (2003) show that the optimum time of forward diffusion can be computed by minimizing the correlation coefficient between the noise (defined as the difference between the original noisy signal and the filtered data) and the signal (filtered data). Their experiments, performed on image intensity values rather than elevation data, show an initial decrease of the correlation coefficient to a minimum value followed by an increase of the correlation coefficient (due to the effect of further filtering on the edges).

We applied this approach to a synthetic landscape with added Gaussian noise and observed that the correlation coefficient between the noise and the filtered signal achieved a minimum value (Figs. S1 and S2 in the Supplementary Information). On the contrary, by applying the same approach to the test landscapes introduced in Section 2 we found that the correlation coefficient between the noise and the filtered signal increased with increasing number of iterations. Further analysis showed that the increase of the correlation coefficient is due to the scale of the noise in the input DEM which is small compared to the signal. As a result, the correlation analysis identified the noise as part of the signal. This behavior was confirmed by adding Gaussian noise to the test landscapes; when the noise is of scale comparable to the scale of the signal, the correlation coefficient decreases with increasing number of iterations (Fig. S3 in the Supplementary Information).

\subsubsection{Surface roughness based analysis of the edge stopping function}

The correlation analysis is not useful to identify the optimum number of iterations over real landscapes, but it suggests that an alternative approach can be developed by analyzing the level of smoothing (or the surface roughness) as a function of number of iterations. If noise is still present in the data, each 
filtering iteration will result in a visible reduction of the surface roughness. An optimal number of iterations can then be identified as the time at which surface roughness does not vary significantly with additional filtering.

Surface roughness can be estimated in several ways (e.g. Fisher et al., 1987; Ritchie, 1995; Rango et al., 2000; McKean and Roering, 2004; Sankey et al., 2010). Here we use the technique of McKean and Roering (2004) and analyze the evolution of the mean and inter quartile range (iqr) of the surface roughness as a function of number of iterations.

At each grid cell (i) a unit vector is defined as: $x_{i}=\sin \theta_{i} \cos \phi_{i}, y_{i}=$ $\sin \theta_{i} \sin \phi_{i}$ and $z_{i}=\cos \theta_{i}$, where $\theta_{i}=90-\operatorname{slope}(\mathrm{deg})$ and $\phi_{i}$ is the aspect. An orientation matrix, $U$, is defined at each cell based on the unit vectors in a three cells by three cells sampling window:

$$
U=\left[\begin{array}{ccc}
\sum x_{i}^{2} & \sum x_{i} y_{i} & \sum x_{i} z_{i} \\
\sum y_{i} x_{i} & \sum y_{i}^{2} & \sum y_{i} z_{i} \\
\sum z_{i} x_{i} & \sum z_{i} y_{i} & \sum z_{i}^{2}
\end{array}\right]
$$

where the sums are computed over the center cell and the eight neighboring cells. The eigenvalues of $U$ provide information about the orientation of the unit vectors (Woodcock, 1977); the ratio of the first $\left(\lambda_{1}\right)$ and second $\left(\lambda_{2}\right)$ eigenvalue is used to describe surface roughness (McKean and Roering, 2004). In smooth landscapes the local vectors have similar orientation, while substantial variability is observed in rough landscapes; the ratio of $\lambda_{1} / \lambda_{2}$ is thus expected to increase as the landscape becomes smoother. Unlike McKean and Roering (2004) we do not analyze the logarithmic ratio of $\lambda_{1}$ and $\lambda_{2}$ and we use the original values.

We analyze surface roughness as a function of number of iterations for both PM1 and PM2 by computing the ratio $\lambda_{1} / \lambda_{2}$ and its mean and iqr (Figure 5). The surface roughness statistics vary with the number of iterations and with the form of the edge stopping function; for PM1 the evolution of roughness characteristics varies among the three landscapes analyzed, while for PM2 the roughness characteristics show consistent behavior. 


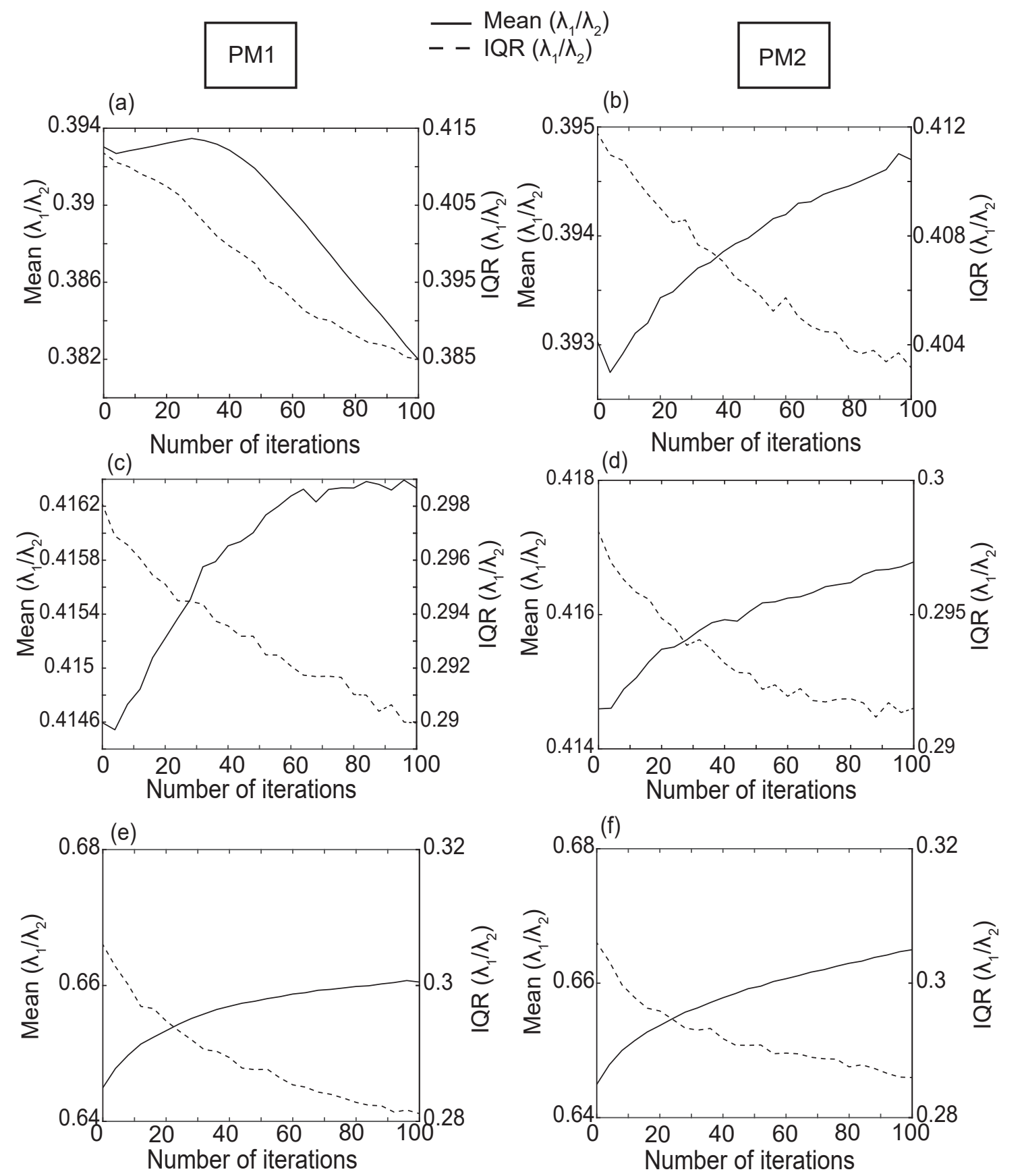

Figure 5: Analysis of the edge stopping functions PM1 and PM2. 
Figure 5: (Continued..) Mean surface roughness (solid lines) and iqr (dashed lines) as a function of number of iterations for a portion of Tennessee Valley, CA (PM1 (a), PM2 (b)), Le Sueur River Basin, MN (PM1 (c), PM2 (d)), and Little Walnut Creek, TX (PM1 (e), PM2 (f)).

PM1 and PM2 respond differently to edges; PM1 prefers sharp contrasts (sharp gradients), while $P M 2$ prefers larger scale contrasts (larger scale features) (Perona and Malik, 1990). For PM1 in the Le Sueur River Basin and Little Walnut Creek the mean ratio increases and becomes constant at $\approx 60$ iterations, while in the Tennessee Valley basin the ratio increases until 30 iterations and then starts decreasing (Figure $5 \mathrm{a}, \mathrm{c}, \mathrm{e}$ ). The iqr of the ratio decreases in all the cases.

With PM2 the mean ratio increases in all the test landscapes, initially at a faster rate, then at a slower rate after $\approx 40$ iterations. The consistent behavior of PM2 across all the landscapes is confirmed by analyzing the contours of the landscapes which after 50 iterations are smooth and devoid of the small scale variability present in the input DEMs (Figs. S4, S5, and S6 in the Supplementary Information).

Given our interest in channel features, rather than sharp structures, the use of PM2 is preferred in both natural and urban landscapes and thus adopted as default edge stopping function in GeoNet. The time of forward diffusion of 50 iterations is also selected as default value given that small scale variability has been sufficiently removed and features of interest enhanced as the roughness statistics and landscape contours show.

\subsection{Identification of likely channelized pixels}

Hillslopes and valleys can be identified from high resolution topography data by comparing the probability density function $(p d f)$ of curvature to the standard Gaussian distribution on a quantile-quantile (qq) plot. The qq-plot indicates whether a sample is drawn from a Gaussian distribution or at what value the pdf of the sample deviates from Normal behavior. Of particular interest for channel extraction is the deviation in the positive tail that marks the transition to valleys (Lashermes et al., 2007), which is automatically detected in GeoNet. Pixels with curvature value above the threshold form the skeleton of likely channelized pixels from which the channel network will be extracted. The identification of the skeleton of likely channelized pixels is further explained in this section, including the curvature definitions implemented in GeoNet and the approach used to compute flow accumulation. 


\subsubsection{Laplacian and geometric curvature}

Two curvature definitions are available in GeoNet. The first option is the Laplacian $\gamma$ defined as the gradient of the elevation gradient $\nabla h$ :

$$
\gamma=\nabla^{2} h
$$

The second option is the geometric curvature $\kappa$ defined as:

$$
\kappa=\nabla \cdot(\nabla h /|\nabla h|)
$$

where the elevation gradient is normalized by its magnitude. The gradients are estimated in GeoNet with a central difference operator, except at the edges where a single-sided difference is used.

The geometric curvature is more effective in identifying convergent features in natural landscapes, while the Laplacian performs better in flat and/or engineered areas where a mixture of natural (channels) and artificial features (ditches and roads) is present (Passalacqua et al., 2012).

The skeleton of likely channelized pixels can be quite noisy due to the possible presence of isolated convergent areas not part of the channel network. Further refinement of the skeleton is achieved through the application of a skeleton thinning parameter applied to the computed flow accumulation as explained below.

\subsubsection{Flow routing and calculation of flow accumulation}

The calculation of flow accumulation in GeoNet starts with the identification of 'sink pixels' as pixels not draining to any location. A water level variable is defined and initialized to the elevation value of each sink pixel. Instead of changing the elevation of each sink pixel, its water level is raised to the lowest elevation in a eight cell neighborhood. A recursive flooding procedure is then used until the water level in the sink pixel is such that it drains to a neighboring cell. The flow is then distributed to the neighboring cells at lower elevation proportionally to slope according to the D8 or Dinfinity methods (Tarboton, 1997), which are both implemented in GeoNet.

Locations along the boundary of the input DEM where the flow leaves the domain are identified as outlet points (Maidment, 2002). The GeoNet flow routing procedure outputs flow accumulation and flow direction at each pixel and an index array that identifies the watershed draining to each outlet point. 


\subsubsection{Skeleton thinning operation}

The skeleton thinning parameter is a user-defined parameter in GeoNet used to remove small convergent areas that are not part of the channel network from the curvature skeleton. The parameter is built on the idea that convergent portions of the landscape that are not part of the network should have small flow accumulation value, smaller than the minimum value at channel heads. The curvature skeleton can then be thinned and 'denoised' by removing the pixels with flow accumulation smaller than the skeleton thinning parameter.

It is important to note that this parameter is not a flow accumulation threshold, but rather a parameter used to simply thin the curvature skeleton from which the channel network will be extracted. As such, the skeleton thinning parameter value should be smaller than the minimum flow accumulation at channel heads to not interfere with channel initiation. Since the minimum value of flow accumulation at channel heads is not known, a reasonable first estimate can be obtained from the slope-area plot of the landscape (Tarboton et al., 1991) along with a visual inspection of the elevation data (Passalacqua and Foufoula-Georgiou, 2015).

\subsection{Extraction of channel heads, channel centerlines, and channel morphol- ogy}

After the operation of nonlinear filtering and the identification of likely channelized pixels, channel heads and channel network are extracted based on geodesic minimization principles. The centerline is then used to 'cut' crosssections perpendicular to it and extract channel morphology properties such as the location of channel banks. The extraction of channel heads, channel centerline, and channel morphology is explained below; we briefly cover the existing portions of the method and focus on the further development of the channel head identification procedure.

\subsubsection{Geodesics and geodesic distance}

Let us assume that channel heads have been identified (as explained later in this section). The feature that connects the channel head to the watershed outlet is the channel itself. We can think of channels as pathways of least cost, or geodesics (Do Carmo, 1976), where the cost $\psi$ is a function of topographic attributes (Passalacqua et al., 2010a):

$$
\psi=\frac{1}{\alpha \cdot A+\delta \cdot C}
$$


where $A$ is the contributing area $\left(L^{2}\right), C$ is the curvature $\left(L / L^{2}\right)$ (either geometric or Laplacian), and $\alpha$ and $\delta$ are constants that handle the dimensionality of Equation 9 ( $\alpha$ has units of $L^{-2}$ and $\delta$ has units of $L$ ). The values of $\alpha$ and $\delta$ are set to $1 \mathrm{~m}^{-2}$ and the mean flow accumulation area, respectively. Passalacqua et al. (2012) showed that adding the skeleton of likely channelized pixel (Skel) is helpful when channels are meandering, resulting in a cost function of the form:

$$
\psi_{\text {new }}=\frac{1}{1 \cdot A+A_{\text {mean }} \cdot S k e l+A_{\text {mean }} \cdot C}
$$

With this cost function, 'shortcuts' of river meanders are penalized forcing channel centerlines to follow the skeleton as much as possible.

After computing the cost function at each location of the landscape, the geodesic distance is calculated from an outlet to any point in the landscape through an operation called geodesic propagation. The geodesic distance is analogous to a travel time between two points. Several algorithms are available for geodesic propagation, including the popular Fast Marching algorithm proposed by Sethian (1996), which is implemented in GeoNet.

\subsubsection{Channel end point identification and search box size}

Channel heads are defined as the most upstream points of erosion and concentrated flow in a channel network (Montgomery and Dietrich, 1988). In GeoNet, these points are identified automatically by scanning the skeleton of likely channelized pixels with a search box that detects the 'end pixel' of each skeleton connected component (connected segments of the skeleton without any breaks) as the pixel with maximum geodesic distance from the watershed outlet (the most upstream point). The skeleton of likely channelized pixels may be disrupted in correspondence of features, such as roads, whose topographic signature does not satisfy the requirements imposed by the curvature threshold and the skeleton thinning parameter. Disruptions may also be due to the presence of natural features such as landslides (Passalacqua et al., 2010b). The search operation through the skeleton thus results in the identification of not only the channel heads, but also the points where channels are disrupted.

The end point search box size was previously a user-defined parameter in GeoNet (Passalacqua and Foufoula-Georgiou, 2015). Here, we propose setting this parameter equal to the median hillslope length computed on the input DEM. This choice is based on the fact that the search box size is 
related to the landscape drainage density: when drainage density is large, the box size should be small (as channels are encountered very often), while when drainage density is small, the box size should be large (as water parcels need to travel longer distances before reaching a channel). It is known that drainage density $\left(D_{d}\right)$ is inversely related to hillslope length $\left(L_{h}\right)$ (Horton, 1932); we can thus inform the choice of the search box size with the characteristics of the channel network through the computation of hillslope lengths. The hillslope length is computed as the distance between each pixel and the first downslope channelized pixel (Tucker et al., 2001) by using the curvature skeleton and the steepest descent information. The median hillslope length is then used as search box size for the identification of channel end points.

We test the effect of this parameter on one of our test landscapes, Tennessee Valley, by analyzing the extracted channel end points (channel heads and channel disruptions) as a function of search box size. The computation of hillslope lengths yields a median value of $56 \mathrm{~m}$ (histogram in Figure 6a). It is our goal to limit the detection of channel disruptions as much as possible and extract the actual channel heads (most upstream end points) in a completely automatic way. A small search box size of $13 m$ (10th quantile of the hillslope length distribution) results in the identification of many channel disruptions (see inset box Figure 6b). A value of $30 \mathrm{~m}$ (25th quantile) ignores most of the disruptions, but a few are still detected (inset box Figure 6c). A box size equal to the median hillslope length of $56 \mathrm{~m}$ (50th quantile) detects mostly channel heads (Figure 6d), while box sizes of $90 \mathrm{~m}$ (75th quantile) and $132 m$ (90th quantile) fail to detect several channel heads (inset boxes Figure $6 e, f)$. 

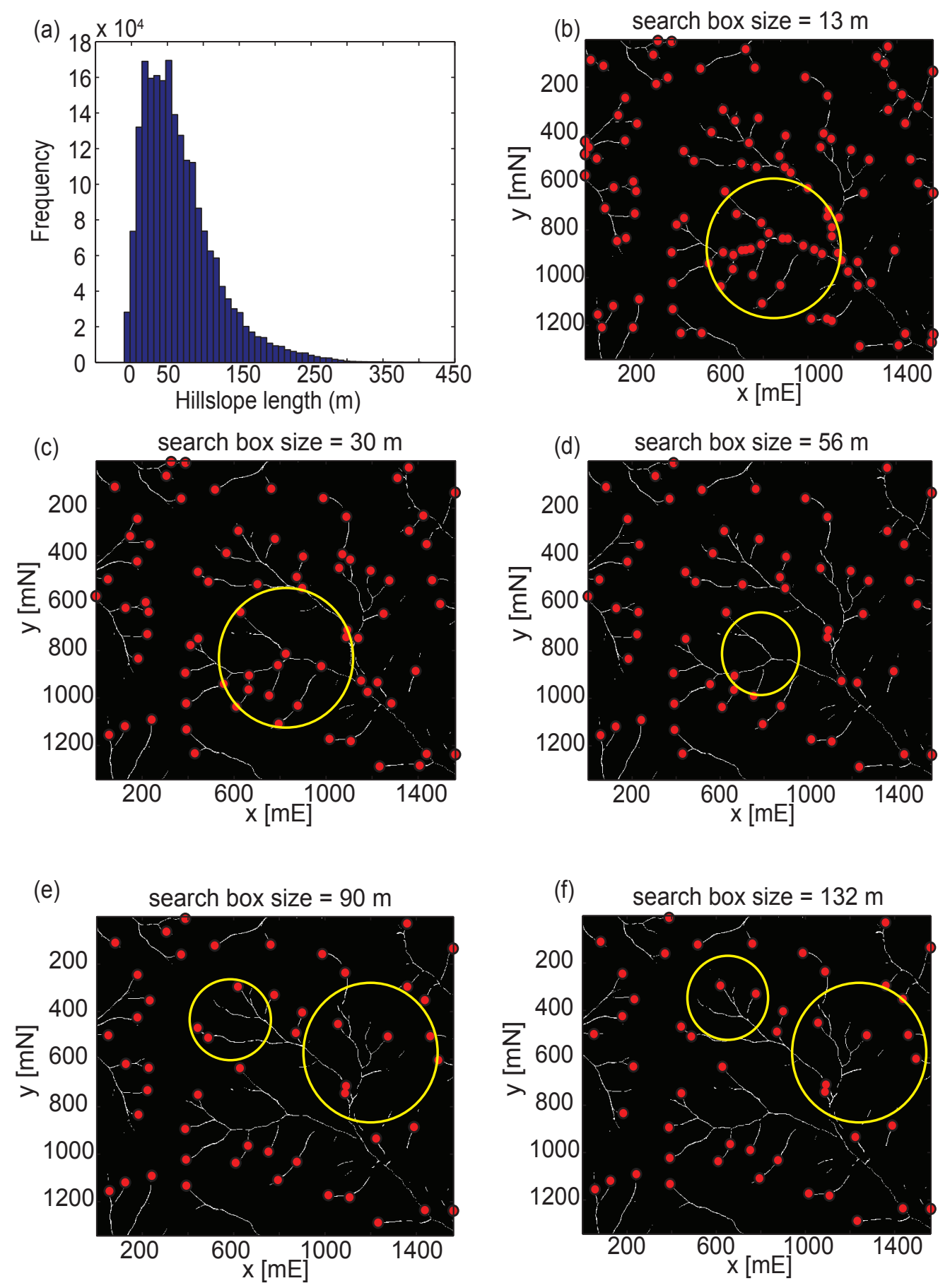

Figure 6: Effect of search box size on end point identification. (a) Histogram of hillslope length for Tennessee Valley. 
Figure 6: (Continued..) Skeleton of likely channelized pixels and detected end points with search box size of (b) $13 \mathrm{~m}$, (c) $30 \mathrm{~m}$, (d) $56 \mathrm{~m}$, (e) $90 \mathrm{~m}$, (f) $132 \mathrm{~m}$.

With the search box size equal to the median hillslope length, GeoNet detects the majority of the field surveyed channel heads. The search box size was previously a user-defined parameter. Based on the analysis here performed, we automatically compute this parameter within GeoNet as the median hillslope length.

\subsubsection{Computation of discrete geodesics and channel network extraction}

Following the channel head identification, a gradient descent on the geodesic distance is used to complete the channel network extraction by tracing channels as paths of minimum cost from each channel end point to the outlet. Channel head locations $(x, y)$ and channel centerlines are saved in shapefile format for visualization in GIS software along with geotiff files of slope, curvature, filtered DEM, skeleton, flow accumulation, and basin and subbasin index arrays.

After extracting the channel network, GeoNet can identify cross-sections, geomorphic bankfull width and water surface elevation, and channel bank locations. The user is referred to Passalacqua et al. (2012) for a detailed description of the channel morphology extraction.

\subsection{Median filter for engineered landscapes}

Engineered landscapes are very challenging for channel extraction methods; convergent artificial features, such as road ditches, are often detected as part of the channel network, leading to the identification of spurious channel heads and inaccurate extraction of the channel network. Passalacqua et al. (2012) proposed a semi-automatic approach that relies on the statistical analysis of Laplacian (equation 7) instead of geometric curvature (equation 8) to favor the detection of channels and exclude artificial features from the extracted channel network. The approach is semi-automatic as it consists of thresholding the curvature at values higher than the threshold automatically detected in the qq-plot.

Here we propose using a low pass filter, specifically the median filter, to automatically remove engineered features such as roads. A low pass filter is commonly used in image processing to remove impulsive random noise, while maintaining important edge features (Lim, 1990). In our application, 
impulsive random noise is represented by features such as road edges and building footprints, while important edge features are channel banks. When a median filter is applied to an image (DEM), a window slides along the intensity (elevation) data; the median elevation value within the window is taken as the output elevation of the pixel being processed. The window size is an important parameter of the median filter usually set with consideration of the average size of the feature of interest. For our application, the features of interest are roads and their average width in USA is $\approx 9-15 m$ (Hughes et al., 2004).

To test the effectiveness of median filtering in removing roads, we apply it to the Le Sueur River Basin (Section 2) which includes natural channels and artificial features (see for example region $A$ in Figure 2 and its 3D and 2D representations in Figure 7a, b). A median filtering operation is performed with window size: $3(9 \mathrm{~m}), 5(15 \mathrm{~m}), 7(21 \mathrm{~m}), 9(27 \mathrm{~m}), 11(33 \mathrm{~m})$, and 13 $(39 \mathrm{~m})$. Note that the DEM resolution is $3 \mathrm{~m}$ in this case and thus window sizes in pixels are multiplied by 3 to obtain the corresponding sizes in metres. With window size of $21 \mathrm{~m}$, median filtering is able to remove most of the road, while keeping channel and valley features well defined (Figure $7 \mathrm{c}, \mathrm{f}, \mathrm{j}$ ). The spike in the along-channel profile corresponding to the road (Figure $7 \mathrm{~d}$ ) is increasingly smoothed as window size increases (Figure 7e-i).

The results show that median filtering with window size of at least $21 \mathrm{~m}$ is able to filter the road features in this landscape. The use of median filtering prior to nonlinear filtering is thus recommended when analyzing engineered landscapes. 

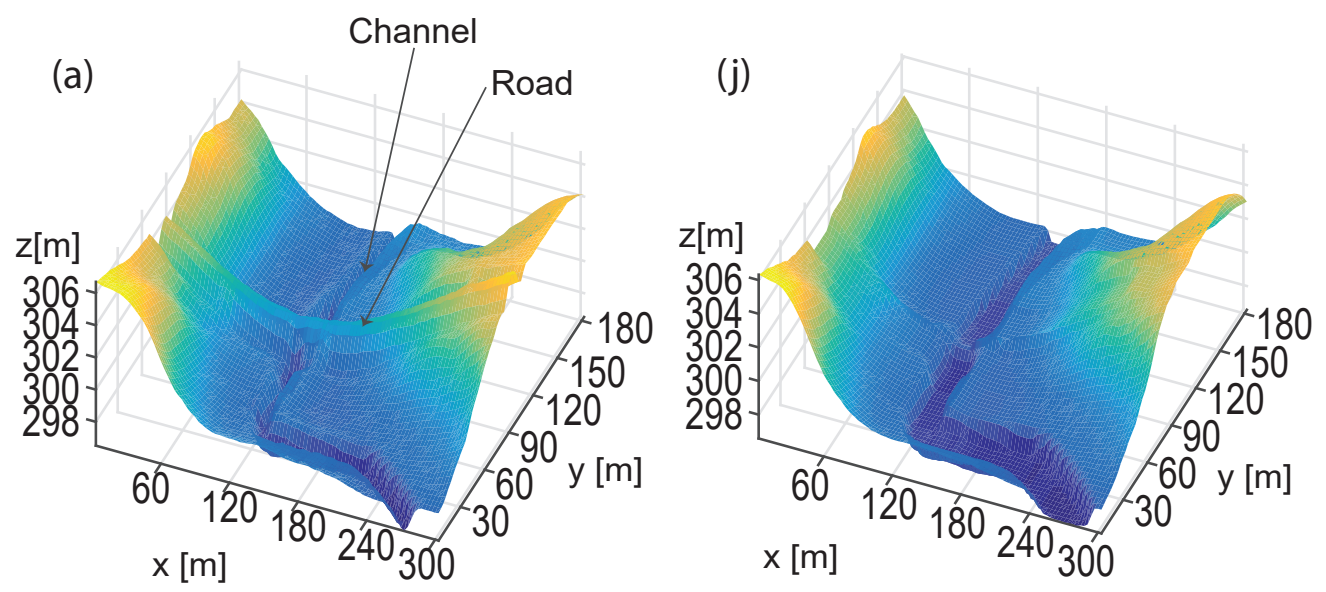

(b)
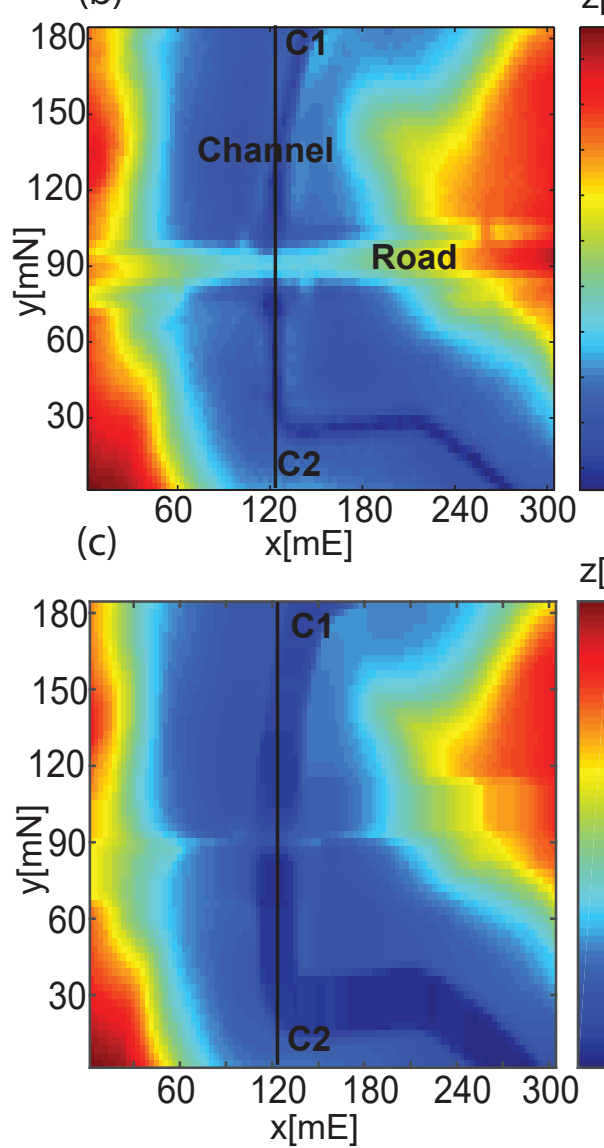

$z[\mathrm{~m}]$

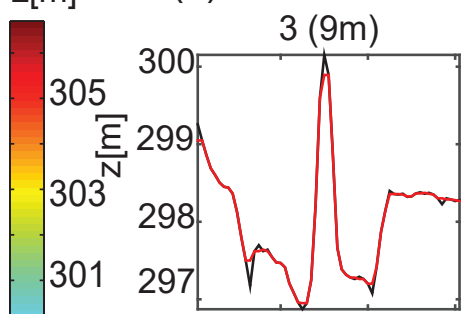

(f) Window size:

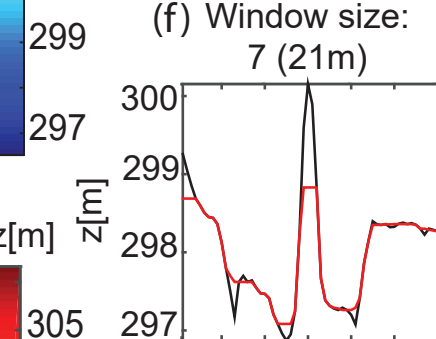

(h) Window size:

303

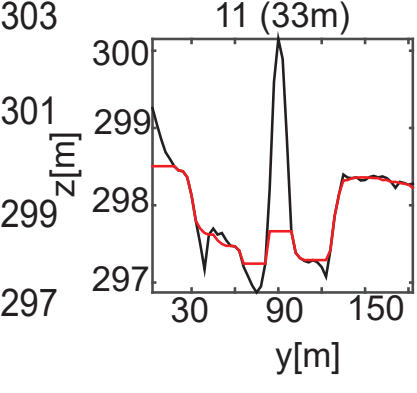

(e) Window size:

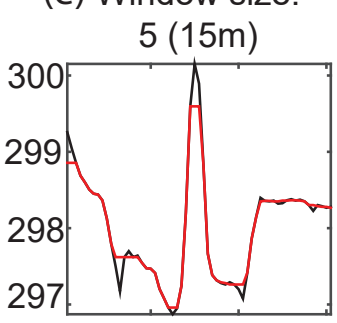

(g) Window size:

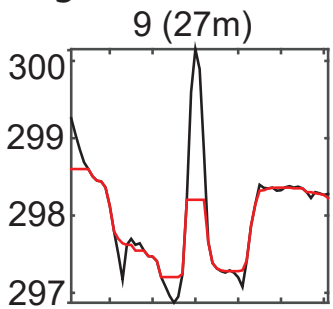

(i) Window size:

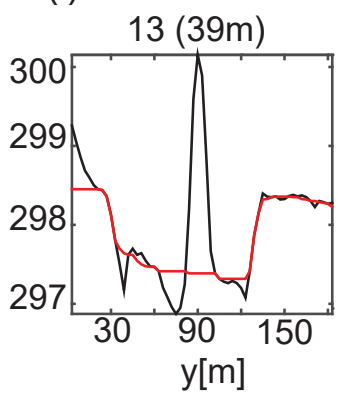

Figure 7: Median filtering for removing roads. Each pixel represents $3 \mathrm{~m}$ in actual scale. 
Figure 7: (Continued..) (a) 3D representation of region $A$ shown in Figure 2a; (b) 2D representation of region $A$; (c) median filtered landscape with window size of $21 \mathrm{~m}$; alongchannel profile of the filtered landscape (red line) with window size of (d) 3 pixels $(9 \mathrm{~m})$, (e) 5 pixels $(15 \mathrm{~m})$, (f) 7 pixels $(21 \mathrm{~m})$, (g) 9 pixels $(27 \mathrm{~m})$, (h) 11 pixels $(33 \mathrm{~m})$, and (i) 13 pixels $(39 \mathrm{~m})$. The profile of the unfiltered landscape (black line) along C1-C2 is shown in all the plots for comparison. (j) $3 \mathrm{D}$ representation of region $A$ after performing a median filter of window size equal to $21 \mathrm{~m}$.

\section{Software development}

GeoNet was originally coded in MATLAB and C programming languages. The software has been further developed to include gdal libraries in MATLAB and has also been coded in Python coupled with GRASS GIS, as described below.

\subsection{MATLAB version of GeoNet}

The MATLAB version of GeoNet employs MATLAB functions included in the Image processing, Statistics, and Mapping Toolbox. To address the needs of users without a Mapping Toolbox license, GeoNet is now coupled with the open source gdal libraries to read and write geotiffs and shapefiles. The flow routing and the Fast Marching algorithms are implemented in $\mathrm{C}$ programming language and accessed from MATLAB via 'mex' functions. Channel heads, channel network, and channel morphology (when extracted) are saved to disk as shapefiles by using the 'shapewrite' function in the Mapping Toolbox. In the gdal version, raster I/O operations are handled via 'mex' function calls to pre-compiled gdal 'read' and 'write' functions.

\subsection{Python version of GeoNet}

The MATLAB version of GeoNet can efficiently process input DEMs up to $\approx 25 \mathrm{~km}^{2}$ at $1 \mathrm{~m}$ resolution. In order to handle larger datasets and remove the dependency on commercial software, GeoNet has been programmed in Python and coupled with GRASS GIS. The libraries NumPy, SciPy, and scikit are used for numerical and scientific computing.

The r.watershed and r.stream.basins GRASS GIS functions (Kinner et al., 2005; Metz et al., 2011; Neteler et al., 2012) are used for computing flow accumulation and identifying the watersheds associated with the outlets of the input DEM, respectively. The r.watershed module does not require pit filling prior to its application because it uses a least-cost algorithm to traverse 
the elevation surface to the outlet. Kinner et al. (2005) showed that this method leads to more accurate results compared to standard methods when applied to high resolution datasets.

The 'disk swap memory' option is used to deal with the cases in which memory requirements exceed the available RAM. The scikit-fmm module is used to calculate the geodesic distance. Channel heads and channel network are saved as shapefiles. GRASS GIS modules are called within GeoNet, thus users are not required to run GRASS GIS.

An analysis of the computational capability of GeoNet is in section 6.4.

\section{Results}

\subsection{Natural landscapes}

The skeleton thinning parameter value used in Tennessee Valley is based on field measurements of flow accumulation at channel heads and set equal to $1100 \mathrm{~m}^{2}$, less than the smallest contributing area recorded at channel heads $\left(\approx 1500 \mathrm{~m}^{2}\right)$ by Montgomery and Dietrich (1989). We compare the extracted network and the field-surveyed data by computing the distance (along channel) between the predicted and the surveyed channel locations, resulting in values of the mean and standard deviation equal to $8.6 \mathrm{~m}$ and $5.3 \mathrm{~m}$, respectively. The surveyed results were mapped by Montgomery and Dietrich (1989) onto an enlarged U.S. Geological Survey Point Bonita 7.5-min quadrangle map with resolution $\approx 10 \mathrm{~m}$. The differences in the extracted and field surveyed channel heads are thus of the same order of magnitude as the resolution of the original field work. Three of the 31 field surveyed channel heads are not identified by GeoNet (A, B, C in Figure 8). A very disrupted signature of these channels is present in the curvature skeleton.

The GeoNet extracted channel network (Figure 8 blue lines) compares well with the digitized field survey channels and channel heads (Figure 8 red lines and green triangles). Differences in channel networks can be expected due to the different resolution of the input dataset and changes in topography during the decade between the field survey and the lidar campaign which may have also included movement of the channel heads. 
(a)

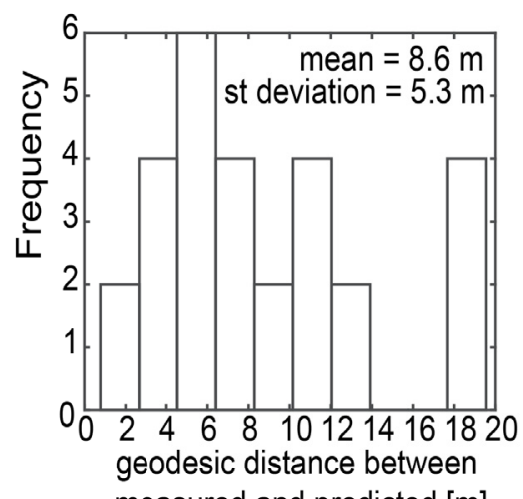

(b) measured and predicted [m]

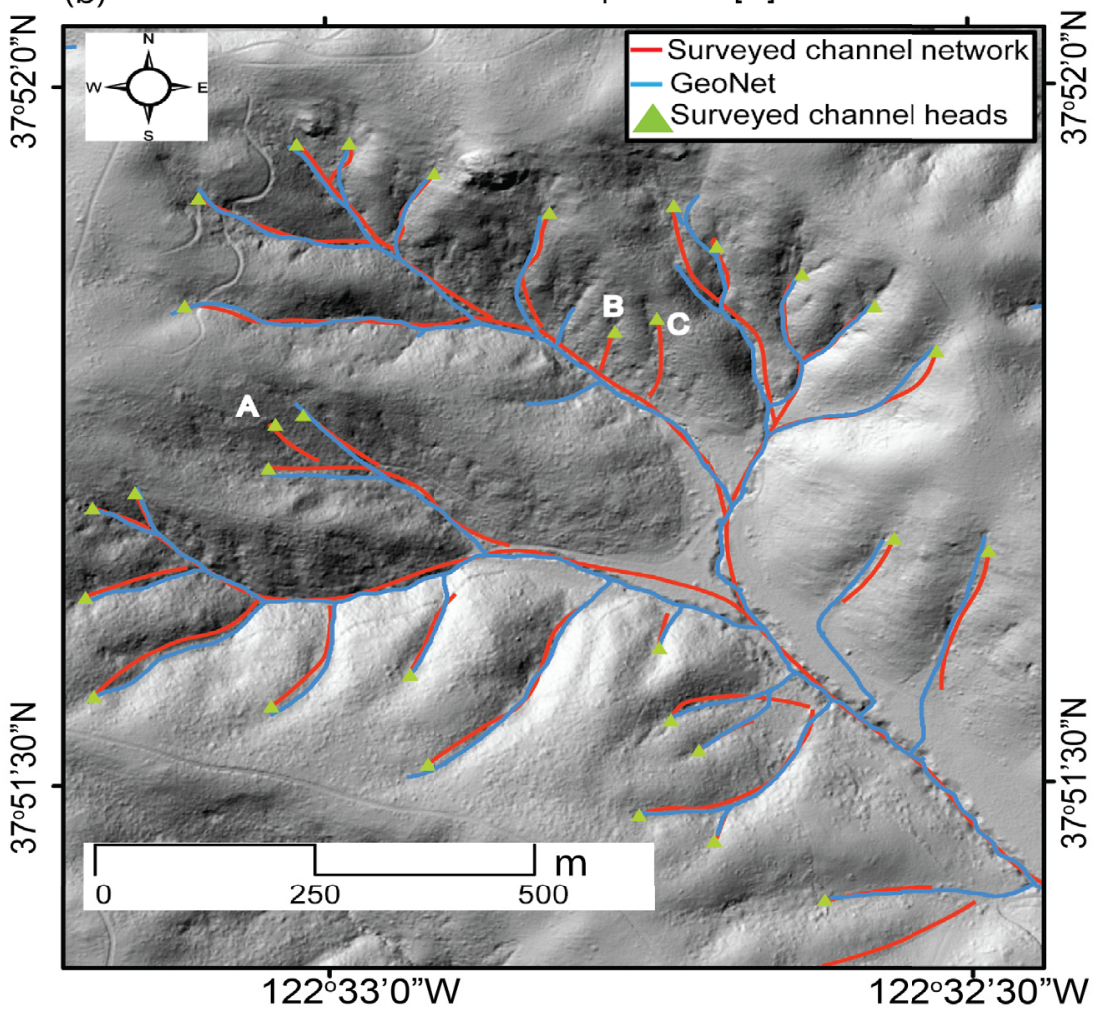

Figure 8: Analysis of GeoNet results in Tennessee Valley, CA. (a) Histogram of distance (absolute value) between mapped and predicted channel heads. Mean and standard deviation of distance are $8.6 \mathrm{~m}$ and $5.3 \mathrm{~m}$ respectively. 
Figure 8: (Continued..) (b) GeoNet extracted channel network and digitized field surveyed channel network and channel heads (Montgomery and Dietrich, 1989). Three of the 31 surveyed channel heads are not identified by GeoNet (A, B, C).

\subsection{Flat and engineered landscapes}

In the Le Sueur River Basin, a low pass median filter with window size of $21 \mathrm{~m}$ is used to remove roads, while preserving the most convergent regions (see also Figure 7).

The skeleton thinning parameter is set to $1500 \mathrm{~m}^{2}$, much less than the channel initiation threshold identified by field studies done in this region (Belmont et al., 2011). Both natural channels (region A in Figure 9) and man made ditches (regions $\mathrm{B}$ and $\mathrm{C}$ ) are detected as part of the channel network with geometric curvature as curvature metric. The use of Laplacian as in Passalacqua et al. (2012) would favor the extraction of natural channels. 

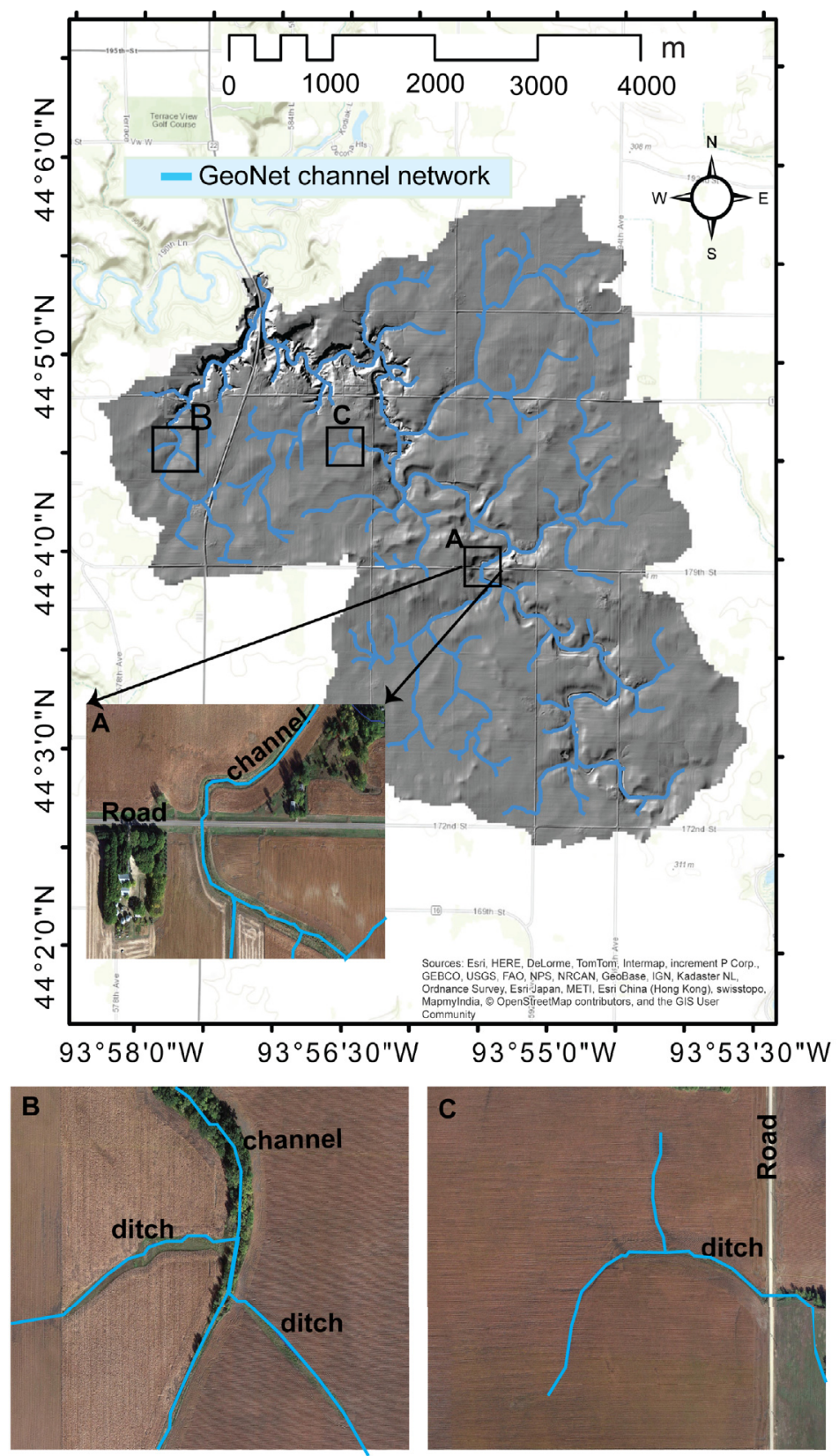

Figure 9: Analysis of GeoNet results in the Le Sueur River Basin, MN. The GeoNet extracted channel network (blue lines) is overlaid on bare earth data. 
Figure 9: (Continued..) Google Earth images highlight locations where the channel crosses a road (A) and ditches are connected to the main channel (B and $\mathrm{C}$ ).

\subsection{Urban landscapes}

We apply GeoNet to an urban landscape with the goal of extracting channels and preferential flow paths active during rainfall events. This is the first time GeoNet is applied to an urban setting. We use a low pass median filter of window size $27 \mathrm{~m}$ and a skeleton thinning parameter equal to $750 \mathrm{~m}^{2}$. Building footprints are used to map building locations as no-data values in the elevation data and thus capture flow paths around buildings. Median filtering and nonlinear filtering are thus performed on the elevation dataset modified by adding no-data values to building locations. This operation guarantees that smoothing and filtering are applied only to the terrain. To allow the computation of the geodesics around buildings, we pass the building footprints as masked values to the Fast Marching module.

Flow directions and flow paths in urban landscapes are affected by manmade structures such as curbs, creeks, and open drains aligned with roads. Typical features occurring in the area analyzed include urban channel heads (Figure 10a, location A1 in Figure 11), drains that resurface from underground (Figure 10b and c, location A2 in Figure 11), culverts (Figure 10d, location A3 in Figure 11), flow paths generated during storm events (Figure 10e), and roads and bridges (Figure 10f, location B in Figure 11).

The effectiveness of GeoNet in dealing with urban environments can be seen, for example, in the three regions $\mathrm{A}, \mathrm{B}$, and $\mathrm{C}$. Region $\mathrm{A}$ includes a channel head (A1 in Figure 11) and part of a drain that goes underground and resurfaces downstream (A2 in Figure 11). The channel centerline extracted by GeoNet (blue line in Figure 11) starts at the drain's initiation point and joins Little Walnut Creek at A3 following the geodesic distance. According to the field reconnaissance, this flow path should merge at location A2 following the underground sewage network path. However, this information is not available in the elevation data and a preferential surface pathway is identified by GeoNet. At location B a drain crosses a road; 'barriers' such as bridges are usually very challenging for channel extraction methods as approaches based, for example, on steepest descent would stop in correspondence of a barrier. The channel extracted by GeoNet is able to pass through the road due to the global minimization principle used in geodesics. Similarly, in region $\mathrm{C}$

the channel passes through a road feature. The use of a mask of building 
footprints guarantees that the extracted channels do not cross any building feature but rather follow preferential flow paths around buildings. 

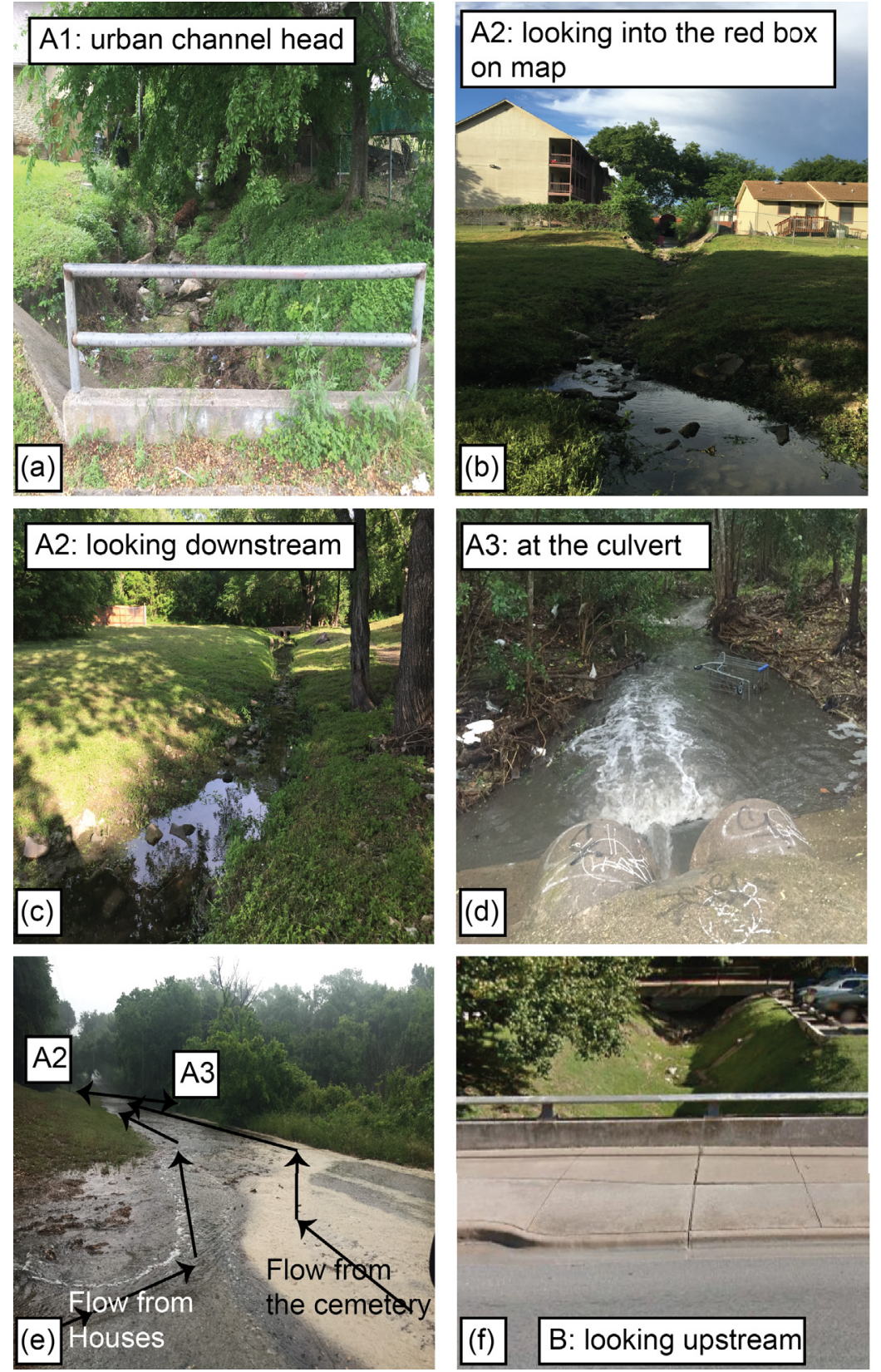

Figure 10: Typical features in urban landscapes. An urban channel head, location A1 (a). A drain resurfaces from underground and joins Little Walnut Creek (image taken looking into the red box A2) (b). Little Walnut Creek (image taken looking downstream from A2) (c). A culvert at A3 in correspondence of a low water crossing (d). Flow paths generated during a rainfall event (e). A drain and the bridge located above it, location B (f). 


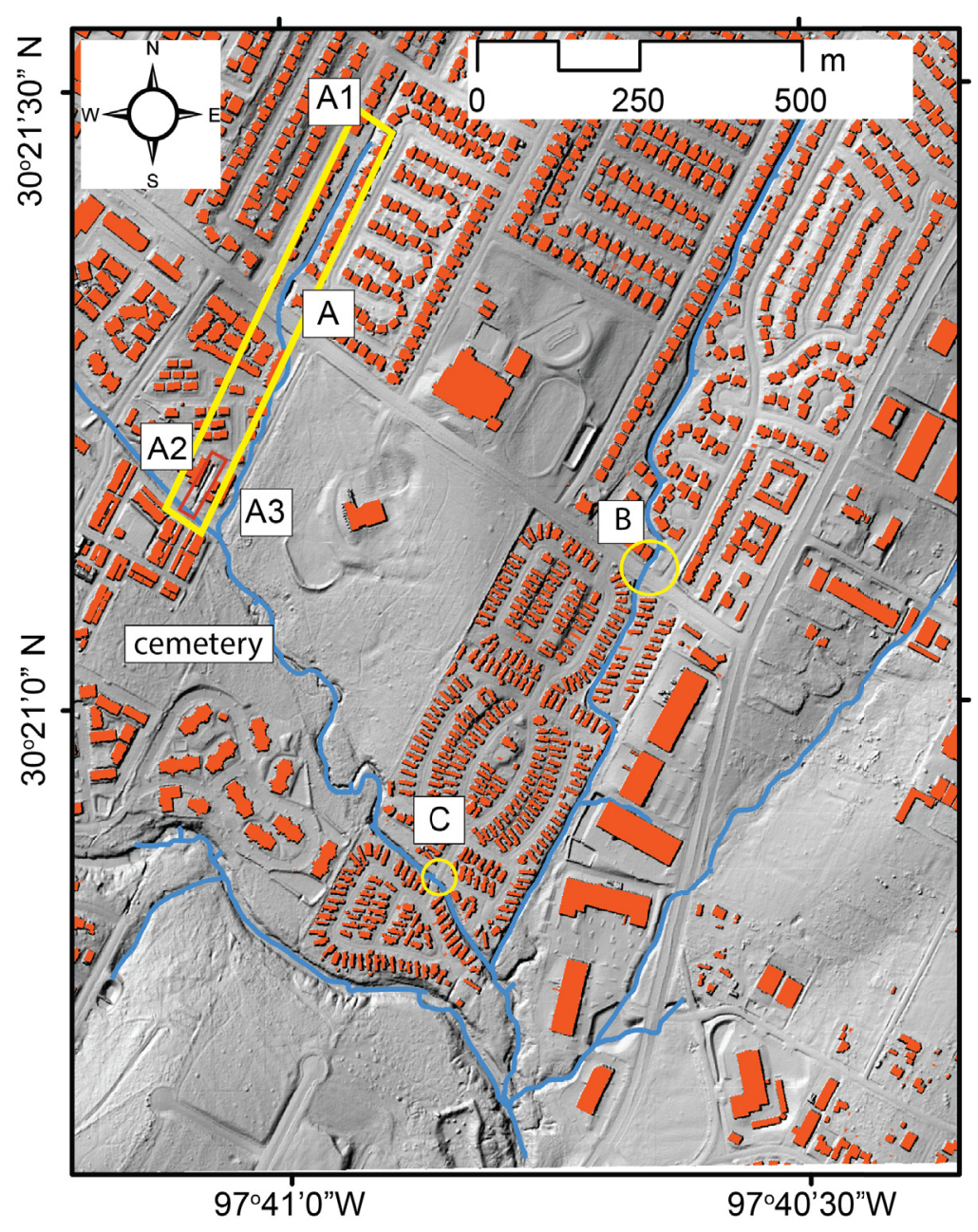

Figure 11: Analysis of GeoNet results in Little Walnut Creek, Travis County, TX. In box A the channel centerline follows a storm water system. A1 marks the location of an urban channel head, A2 the location where the drain resurfaces from underground (red box), and A3 the location of a culvert (see also corresponding photos in Figure 10). B and C mark locations where GeoNet handles the presence of bridges without manual modification of the elevation data. The channel passes through such disruptions following the global minimization of the cost function.

\subsection{Large scale applications}

We analyze the capability of GeoNet of handling large datasets by reporting the time to extraction completion against the size of the input DEM (number of pixels). All the datasets (listed with their resolution and size in Table 1) 
were run on a desktop machine with Intel Xenon processor (X5647) running at $2.93 \mathrm{GHz}, 12 \mathrm{~GB}$ RAM, with a 64 bit Windows 7 operating system. A 64 bit Python 2.7 version and GRASS GIS 7.0 stable release for Windows were used. All the input datasets were bare-earth high resolution topography DEMs.

The MATLAB version of GeoNet can process watersheds of the size of the Ikawa (dataset B in Figure 12$)\left(\approx 25 \mathrm{~km}^{2}\right)$ in around 40 minutes. Datasets larger than $\approx 25 \mathrm{~km}^{2}$ take longer than 80 minutes due to computational expenses in the flow accumulation, Fast Marching, and compute discrete geodesics modules. The Python version of GeoNet overcomes this problem by integrating GRASS GIS modules into GeoNet and can completely process the Ikawa watershed in $\approx 13$ minutes.

As the dataset size increases, the time to completion of GeoNet (Python version) increases from $\approx 4.8$ minutes for the Loch Vale watershed to $\approx$ 84 minutes for the entire Santa Catalina Mountains (Figure 12). We note that even if large watersheds, such as the Santa Catalina Mountains, can be efficiently run in GeoNet, terrain characteristics can vary substantially over such a large spatial extent. More accurate results are obtained applying GeoNet to smaller sub-areas.

For small datasets, the execution times of the MATLAB and Python version of GeoNet are similar and thus the choice of one version versus the other depends on the user's preference. While MATLAB provides a quick user-friendly environment to test and run GeoNet, Python provides the capability of analyzing larger datasets in less time. 


\begin{tabular}{|c|c|c|c|c|}
\hline Label & Dataset Name & $\begin{array}{l}\text { DEM pixel } \\
\text { resolution } \\
\text { (m) }\end{array}$ & $\begin{array}{l}\text { Number of } \\
\text { pixels }\end{array}$ & $\begin{array}{l}\text { Time } \\
\text { (minutes) }\end{array}$ \\
\hline A & $\begin{array}{l}\text { Loch Vale Watershed, } \\
\text { CO, USA }\end{array}$ & 1 & $11.9 \cdot 10^{6}$ & 4.82 \\
\hline B & Ikawa Watershed, Japan & 1 & $22.5 \cdot 10^{6}$ & 13.05 \\
\hline $\mathrm{C}$ & $\begin{array}{l}\text { The Reynolds } \text { Creek } \\
\text { CZO, ID, USA }\end{array}$ & 3 & $47 \cdot 10^{6}$ & 26.38 \\
\hline $\mathrm{D}$ & $\begin{array}{l}\text { Mission Creek water- } \\
\text { shed, Australia }\end{array}$ & 1 & $64.7 \cdot 10^{6}$ & 60.42 \\
\hline $\mathrm{E}$ & $\begin{array}{l}\text { Santa Catalina Moun- } \\
\text { tains, AZ, USA }\end{array}$ & 2 & $114 \cdot 10^{6}$ & 83.697 \\
\hline
\end{tabular}

Table 1: List of datasets and their characteristics used in the large scale application analysis.

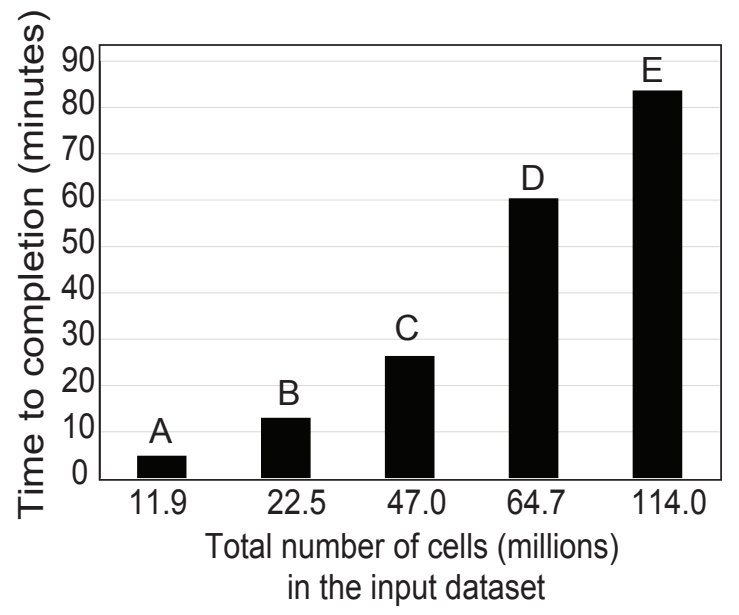

Figure 12: The CPU time of completion of GeoNet (Python version) versus dataset size (number of pixels). The letters A, B, C, D, and $\mathrm{E}$ refer to the test datasets listed in Table 1. Note that these results were obtained using a single processor.

\section{Discussion and Conclusions}

High resolution topography data capture channel networks at the correct drainage density. The extraction of these features requires the development and use of tools that do not impose a strict flow accumulation threshold. 
One of these methods is GeoNet which combines filtering, statistical analysis of topographic attributes, and geodesic minimization principles to extract channel networks, channel heads, and channel morphology in an automatic and objective manner.

In this work, we have further developed the GeoNet method and software, with particular focus on aspects of the GeoNet method that had not been previously analyzed: the edge stopping function and the number of iterations used in the nonlinear filtering operation, the search box size for the identification of channel heads, and the extraction of flow paths in urban landscapes. Additionally, the software has been further developed to handle larger datasets than previously analyzed and remove the dependency on commercial software.

The two edge stopping functions PM1 and PM2 were compared by analyzing surface roughness statistics as a function of number of iterations. The analysis showed that PM2 does not depend on landscape characteristics, while PM1 shows a different response in natural versus engineered landscapes. We thus confirmed the use of $P M 2$ as default edge stopping function. The surface roughness metric (mean ratio of the first two eigenvalues of the orientation matrix) increased with increasing number of iterations as expected. The default number of iterations was confirmed to 50 for the analysis of any landscape based on the rate of increase of the mean ratio and on the landscape contours.

The channel head identification procedure was improved by computing hillslope lengths within GeoNet and setting the parameter search box size equal to their median value. This operation allows the automatic definition of the parameter, previously set by the users. An analysis with search box size equal to increasing quantiles of the hillslope length distribution showed that the median value achieves the best compromise between a box too small, which results in the identification of many skeleton disruptions as channel end points, and a box too large which may not detect several channel heads close to each other.

Roads and bridges challenge channel extraction methods as any feature crossing a channel acts as a barrier to the steepest descent method. The geodesics approach used in GeoNet overcomes this problem by performing a global minimization of the cost function; the extraction of the channel centerline is not affected by local disruptions. Additionally, engineered landscapes are characterized by the presence of both natural and artificial convergent features, resulting in the detection of roads ditches as part of the channel 
network. In order to favor the extraction of natural channels, Passalacqua et al. (2012) proposed a semi-automatic approach that consisted of manually thresholding the Laplacian curvature. Here we proposed the use of a low pass median filter able to remove impulsive noise. The window size of the median filter was set equal to the width of the road features. We note that the removal of roads is not performed to enable the extraction of continuous channels through disruptions as the geodesic approach is capable of doing that. The median filter is adopted instead to help reduce the detection of convergent man-made features in the landscape, such as road ditches.

The application of GeoNet to three test landscapes showed the ability of the method to accurately extract flow paths over different terrains. In Tennessee Valley, GeoNet identified the majority of the surveyed channel heads, with some differences likely due to data resolution and changes in the landscape over time. In the Le Sueur River basin, the combination of median filtering and geometric curvature resulted in the detection of both channels and drainage ditches. The first application of GeoNet in an urban watershed, Little Walnut Creek, showed that the method is able to accurately trace flow paths in urban environments, which is fundamental to improving hydraulic modeling and the prediction of flooding extent.

The change in programming environment from MATLAB to the open source Python language removed the dependency on commercial licensing and increased the capability of GeoNet of handling large datasets. The memory constraints in processing large datasets within MATLAB are alleviated by the Python programming environment and the integration with GRASS GIS.

There are several ways in which the GeoNet method and software can be further improved. For example, recently developed flow direction estimation methods, such as D8-LTD (Orlandini et al., 2003, 2014) could be easily incorporated in the tool. The dependence on the user defined skeleton thinning parameter could potentially be removed by employing a filtering approach that relies on a weighted flow accumulation (Peucker and Douglas, 1975; Band, 1986).

Increased computational efficiency could be achieved by parallelizing several functions of the GeoNet work-flow with an approach similar to the one used in TauDEM (Tarboton and Ames, 2001; Tesfa et al., 2011) or by applying GeoNet in smaller sub-areas and then combining the extraction results. The latter approach would require the capability of ensuring network connectivity among the different sub-areas. 


\section{Acknowledgments}

The authors gratefully acknowledge support from the National Science Foundation (grants GSS/BCS 1063228, CAREER/EAR-1350336, and FESD/EAR1135427). HS was partly supported by a USGS John Wesley Powell Center for Analysis and Synthesis as Fellow of the Powell Center Working Group on High Resolution Topography. GeoNet is available for download at https: //sites.google.com/site/geonethome/source-code. We thank the Editor, Daniel Ames, and the three reviewers David Tarboton, Stefano Orlandini, and Paolo Tarolli for their comments which have helped improve this manuscript. 


\section{References}

Band, L. E., 1986. Topographic partition of watersheds with digital elevation models. Water Resources Research 22 (1), 15-24.

Belmont, P., Gran, K., Jennings, C. E., Wittkop, C., Day, S. S., 2011. Holocene landscape evolution and erosional processes in the le sueur river, central minnesota. Field Guides 24, 439-455.

Bhowmik, A. K., Metz, M., Schäfer, R. B., 2015. An automated, objective and open source tool for stream threshold selection and upstream riparian corridor delineation. Environmental Modelling \& Software 63, 240-250.

Burt, P. J., Adelson, E. H., 1983. The laplacian pyramid as a compact image code. Communications, IEEE Transactions on 31 (4), 532-540.

Catté, F., Lions, P.-L., Morel, J.-M., Coll, T., 1992. Image selective smoothing and edge detection by nonlinear diffusion. SIAM Journal on Numerical Analysis 29 (1), 182-193.

Cazorzi, F., Fontana, G. D., Luca, A. D., Sofia, G., Tarolli, P., 2013. Drainage network detection and assessment of network storage capacity in agrarian landscape. Hydrological Processes 27 (4), 541-553.

Costacabral, M., Burges, S., 1994. Digital elevation model networks (demon) - a model of flow over hillslopes for computation of contributing and dispersal areas. Water Resources Research 30 (6), 1681-1692.

Dietrich, W. E., Wilson, C. J., Montgomery, D. R., McKean, J., 1993. Analysis of erosion thresholds, channel networks, and landscape morphology using a digital terrain model. The Journal of Geology 101 (2), pp. 259278.

URL http://www.jstor.org/stable/30081151

Do Carmo, M. P., 1976. Differential geometry of curves and surfaces. Vol. 2. Prentice-hall Englewood Cliffs.

Fisher, N. I., Lewis, T., Embleton, B. J., 1987. Statistical analysis of spherical data. Cambridge university press. 
Giannoni, F., Roth, G., Rudari, R., JUN 2005. A procedure for drainage network identification from geomorphology and its application to the prediction of the hydrologic response. Advances in Water Resources 28 (6), $567-581$.

Gilboa, G., Zeevi, Y. Y., Sochen, N. A., 2001. Complex difusion processes for image filtering. In: Scale-Space and Morphology in Computer Vision. Springer, pp. 299-307.

Glennie, C. L., Carter, W. E., Shrestha, R. L., Dietrich, W. E., 2013. Geodetic imaging with airborne lidar: the earth's surface revealed. Reports on Progress in Physics 76 (8), 086801.

URL http://stacks.iop.org/0034-4885/76/i=8/a=086801

Gran, K. B., Belmont, P., Day, S. S., Finnegan, N., Jennings, C., Lauer, J. W., Wilcock, P. R., 2011. Landscape evolution in south-central minnesota and the role of geomorphic history on modern erosional processes. GSA Today 21 (9), 7-9.

Gran, K. B., Belmont, P., Day, S. S., Jennings, C., Johnson, A., Perg, L., Wilcock, P. R., 2009. Geomorphic evolution of the le sueur river, minnesota, usa, and implications for current sediment loading. Geological Society of America Special Papers 451, 119-130.

Hancock, G., Evans, K., 2006. Channel head location and characteristics using digital elevation models. Earth Surface Processes and Landforms $31(7), 809-824$.

Harpold, A. A., Marshall, J. A., Lyon, S. W., Barnhart, T. B., Fisher, B., Donovan, M., Brubaker, K. M., Crosby, C. J., Glenn, N. F., Glennie, C. L., Kirchner, P. B., Lam, N., Mankoff, K. D., McCreight, J. L., Molotch, N. P., Musselman, K. N., Pelletier, J., Russo, T., Sangireddy, H., Sjöberg, Y., Swetnam, T., West, N., 2015. Laser vision: lidar as a transformative tool to advance critical zone science. Hydrology and Earth System Sciences Discussions 12 (1), 1017-1058.

URL http://www.hydrol-earth-syst-sci-discuss.net/12/1017/ 2015/

Horton, R. E., 1932. Drainage-basin characteristics. Eos, Transactions American Geophysical Union 13 (1), 350-361. 
Hughes, W. E., Eccles, K., Harwood, D., Potts, I., Hauer, E., 2004. Development of a Highway Safety Manual. Transportation Research Board.

Jones, N. L., Wright, S. G., Maidment, D. R., 1990. Watershed delineation with triangle-based terrain models. Journal of Hydraulic Engineering 116 (10), 1232-1251.

Kinner, D., Mitasova, H., Harmon, R., Toma, L., Stallard, R., 2005. Gisbased stream network analysis for the chagres river basin, republic of panama. The Rio Chagres: A multidisciplinary profile of a tropical watershed, Springer/Kluwer, 83-95.

Koenderink, J. J., 1984. The structure of images. Biological Cybernetics $50(5), 363-370$.

Lacroix, M. P., Martz, L. W., Kite, G. W., Garbrecht, J., 2002. Using digital terrain analysis modeling techniques for the parameterization of a hydrologic model. Environmental Modelling \& Software 17 (2), 125-134.

Lashermes, B., Foufoula-Georgiou, E., Dietrich, W. E., 2007. Channel network extraction from high resolution topography using wavelets. Geophysical Research Letters 34 (23).

Lim, J. S., 1990. Two-dimensional signal and image processing. Englewood Cliffs, NJ, Prentice Hall, 1990, 710 p. 1.

Maidment, D. R., 2002. Arc Hydro: GIS for water resources. Vol. 1. ESRI, Inc.

McCullagh, P., 1978. Modern concepts in geomorphology. Oxford University Press.

McKean, J., Roering, J., 2004. Objective landslide detection and surface morphology mapping using high-resolution airborne laser altimetry. Geomorphology 57 (3), 331-351.

McNamara, J. P., Ziegler, A. D., Wood, S. H., Vogler, J. B., 2006. Channel head locations with respect to geomorphologic thresholds derived from a digital elevation model: A case study in northern thailand. Forest Ecology and Management 224 (1), 147-156. 
Metz, M., Mitasova, H., Harmon, R., 2011. Efficient extraction of drainage networks from massive, radar-based elevation models with least cost path search. Hydrology and Earth System Sciences 15 (2), 667-678.

Montgomery, D. R., Dietrich, W. E., 1988. Where do channels begin? Nature 336 (6196), 232-234.

Montgomery, D. R., Dietrich, W. E., 1989. Source areas, drainage density, and channel initiation. Water Resources Research 25 (8), 1907-1918.

Montgomery, D. R., Foufoula-Georgiou, E., 1993. Channel network source representation using digital elevation models. Water Resources Research 29 (12), 3925-3934.

Moretti, G., Orlandini, S., 2008. Automatic delineation of drainage basins from contour elevation data using skeleton construction techniques. Water Resources Research 44 (5).

Mrázek, P., Navara, M., 2003. Selection of optimal stopping time for nonlinear diffusion filtering. International Journal of Computer Vision 52 (2-3), 189-203.

Nelson, E. J., Jones, N. L., Miller, A. W., 1994. Algorithm for precise drainage-basin delineation. Journal of Hydraulic Engineering 120 (3), 298312 .

Neteler, M., Bowman, M. H., Landa, M., Metz, M., 2012. \{GRASS\} gis: A multi-purpose open source \{GIS\}. Environmental Modelling \& Software $31(0), 124-130$.

URL http://www.sciencedirect.com/science/article/pii/ S1364815211002775

Orlandini, S., Moretti, G., Franchini, M., Aldighieri, B., Testa, B., 2003. Path-based methods for the determination of nondispersive drainage directions in grid-based digital elevation models. Water Resources Research 39 (6).

Orlandini, S., Moretti, G., Gavioli, A., 2014. Analytical basis for determining slope lines in grid digital elevation models. Water Resources Research 50 (1), 526-539. 
Orlandini, S., Tarolli, P., Moretti, G., Dalla Fontana, G., 2011. On the prediction of channel heads in a complex alpine terrain using gridded elevation data. Water Resources Research 47 (2).

Passalacqua, P., Belmont, P., Foufoula-Georgiou, E., 2012. Automatic geomorphic feature extraction from lidar in flat and engineered landscapes. Water Resources Research 48 (3), n/a-n/a.

URL http://dx.doi.org/10.1029/2011WR010958

Passalacqua, P., Belmont, P., Staley, D. M., Simley, J. D., Arrowsmith, J. R., Bode, C. A., Crosby, C., DeLong, S. B., Glenn, N. F., Kelly, S. A., et al., 2015. Analyzing high resolution topography for advancing the understanding of mass and energy transfer through landscapes: A review. Earth-Science Reviews 148, 174-193.

Passalacqua, P., Do Trung, T., Foufoula-Georgiou, E., Sapiro, G., Dietrich, W. E., 2010a. A geometric framework for channel network extraction from lidar: Nonlinear diffusion and geodesic paths. Journal of Geophysical Research: Earth Surface (2003-2012) 115 (F1).

Passalacqua, P., Foufoula-Georgiou, E., 2015. Comment on objective extraction of channel heads from high-resolution topographic data by fiona $\mathrm{j}$. clubb et al. Water Resources Research.

URL http://dx.doi.org/10.1002/2014WR016412

Passalacqua, P., Hillier, J., Tarolli, P., 2014. Innovative analysis and use of high-resolution dtms for quantitative interrogation of earth-surface processes. Earth Surface Processes and Landforms 39 (10), 1400-1403.

Passalacqua, P., Tarolli, P., Foufoula-Georgiou, E., 2010b. Testing spacescale methodologies for automatic geomorphic feature extraction from lidar in a complex mountainous landscape. Water Resources Research 46 (11).

Pelletier, J. D., 2013. A robust, two-parameter method for the extraction of drainage networks from high-resolution digital elevation models (dems): Evaluation using synthetic and real-world dems. Water Resources Research 49 (1), 75-89.

Perona, P., Malik, J., 1990. Scale-space and edge detection using anisotropic diffusion. Pattern Analysis and Machine Intelligence, IEEE Transactions on $12(7), 629-639$. 
Peucker, T. K., Douglas, D. H., 1975. Detection of surface-specific points by local parallel processing of discrete terrain elevation data. Computer Graphics and Image Processing 4 (4), 375-387.

Rango, A., Chopping, M., Ritchie, J., Havstad, K., Kustas, W., Schmugge, T., 2000. Morphological characteristics of shrub coppice dunes in desert grasslands of southern new mexico derived from scanning lidar. Remote Sensing of Environment 74 (1), 26-44.

Ritchie, J. C., 1995. Airborne laser altimeter measurements of landscape topography. Remote Sensing of Environment 53 (2), 91-96.

Sankey, J. B., Glenn, N. F., Germino, M. J., Gironella, A. I. N., Thackray, G. D., 2010. Relationships of aeolian erosion and deposition with lidar-derived landscape surface roughness following wildfire. Geomorphology 119 (1), 135-145.

Sethian, J. A., 1996. A fast marching level set method for monotonically advancing fronts. Proceedings of the National Academy of Sciences 93 (4), $1591-1595$.

Sofia, G., Marinello, F., Tarolli, P., 2014a. A new landscape metric for the identification of terraced sites: the slope local length of auto-correlation (sllac). ISPRS Journal of Photogrammetry and Remote Sensing 96, 123133.

Sofia, G., Prosdocimi, M., Dalla Fontana, G., Tarolli, P., 2014b. Modification of artificial drainage networks during the past half-century: Evidence and effects in a reclamation area in the veneto floodplain (italy). Anthropocene $6,48-62$.

Sofia, G., Tarolli, P., Cazorzi, F., Dalla Fontana, G., 2011. An objective approach for feature extraction: distribution analysis and statistical descriptors for scale choice and channel network identification. Hydrology and Earth System Sciences 15 (5), 1387-1402.

Tarboton, D. G., 1997. A new method for the determination of flow directions and upslope areas in grid digital elevation models. Water Resources Research 33 (2), 309-319. 
Tarboton, D. G., Ames, D. P., 2001. Advances in the mapping of flow networks from digital elevation data. In: World Water and Environmental Resources Congress. Am. Soc Civil Engrs USA, pp. 20-24.

Tarboton, D. G., Bras, R. L., Rodriguez-Iturbe, I., 1988. The fractal nature of river networks. Water Resources Research 24 (8), 1317-1322.

Tarboton, D. G., Bras, R. L., Rodriguez-Iturbe, I., 1991. On the extraction of channel networks from digital elevation data. Hydrological Processes 5 (1), $81-100$.

Tarolli, P., 2014. High-resolution topography for understanding earth surface processes: opportunities and challenges. Geomorphology 216, 295-312.

Tarolli, P., Dalla Fontana, G., 2009. Hillslope-to-valley transition morphology: new opportunities from high resolution dtms. Geomorphology 113 (1), $47-56$.

Tesfa, T. K., Tarboton, D. G., Watson, D. W., Schreuders, K. A., Baker, M. E., Wallace, R. M., 2011. Extraction of hydrological proximity measures from dems using parallel processing. Environmental Modelling \& Software 26 (12), 1696-1709.

Tucker, G. E., Catani, F., Rinaldo, A., Bras, R. L., 2001. Statistical analysis of drainage density from digital terrain data. Geomorphology 36 (3), 187202.

Vivoni, E. R., Ivanov, V. Y., Bras, R. L., Entekhabi, D., 2004. Generation of triangulated irregular networks based on hydrological similarity. Journal of Hydrologic Engineering 9 (4), 288-302.

Weickert, J., 1997. A review of nonlinear diffusion filtering. In: Scale-space theory in computer vision. Springer, pp. 1-28.

Wiener, N., 1949. Extrapolation, interpolation, and smoothing of stationary time series, with engineering applications. 1949.

Witkin, A. P., 1984. Scale-space filtering: A new approach to multi-scale description. In: Acoustics, Speech, and Signal Processing, IEEE International Conference on ICASSP'84. Vol. 9. IEEE, pp. 150-153. 
Woodcock, N., 1977. Specification of fabric shapes using an eigenvalue method. Geological Society of America Bulletin 88 (9), 1231-1236.

Yang, P., Ames, D. P., Fonseca, A., Anderson, D., Shrestha, R., Glenn, N. F., Cao, Y., 2014. What is the effect of lidar-derived dem resolution on largescale watershed model results? Environmental Modelling \& Software 58, $48-57$.

Zhou, Q., Pilesjö, P., Chen, Y., 2011. Estimating surface flow paths on a digital elevation model using a triangular facet network. Water Resources Research 47 (7). 